\title{
Linear multispecies gyrokinetic flux tube benchmarks in shaped tokamak plasmas
}

G. Merlo, ${ }^{1}$ O. Sauter, ${ }^{1}$ S. Brunner, ${ }^{1}$ A. Burckel, ${ }^{1}$ Y. Camenen, ${ }^{2}$ F.J. Casson, ${ }^{3}$ W.

Dorland, ${ }^{4}$ E. Fable, ${ }^{5}$ T. Görler, ${ }^{5}$ F. Jenko, ${ }^{6}$ A.G. Peeters, ${ }^{7}$ D. Told ${ }^{6}$ and L. Villard ${ }^{1}$

1) Ecole Polytechnique Fédérale de Lausanne (EPFL), Swiss Plasma Center (SPC), CH-1015 Lausanne, Switzerland

2) Aix-Marseille Université CNRS, PIIM UMR 7345, 13397, Marseille,

France

3) CCFE, Culham Science Centre, Abingdon, Oxon, OX14 3DB, $U K$

4) Department of Physics, University of Maryland, College Park, Maryland 20742, $U S A$

5) Max-Planck Institut für Plasmaphysik, Boltzmannstr. 2, D-85748 Garching, Germany

6) Department of Physics \& Astronomy, University of California, Los Angeles, California 90095, USA

7) Physics Department, University of Bayreuth, 95440 Bayreuth, Germany

(Dated: January 26, 2016) 
Verification is the fundamental step that any turbulence simulation code has to be submitted to in order to assess the proper implementation of the underlying equations. We have carried out a cross comparison of three flux tube gyrokinetic codes, GENE [F. Jenko et al., Phys.Plasmas 7,1904 (2000)], GKW [A.G. Peeters et al., Comput.Phys.Commun, 180, 7053 (2009)] and GS2 [W. Dorland et al., Phys.Rev.Lett. 85, 5579 (2000)], focusing our attention on the effect of realistic geometries described by a series of MHD equilibria with increasing shaping complexity. To simplify the effort, the benchmark has been limited to the electrostatic collisionless linear behaviour of the system. A fully gyokinetic model has been used to describe the dynamics of both ions and electrons. Several tests have been carried out looking at linear stability at ion and electron scales, where for the assumed profiles ITG/TEM and ETG modes are unstable. The capability of the codes to handle a non-zero ballooning angle has been successfully benchmarked in the ITG regime. Finally, the standard RosenbluthHinton test has been successfully carried out looking at the effect of shaping on Zonal Flows (ZFs) and Geodesic Acoustic Modes (GAMs). Inter-code comparison as well as validation of simulation results against analytical estimates have been accomplished. All the performed tests confirm that plasma elongation strongly stabilizes plasma instabilities as well as leads to a strong increase in ZF residual and GAM damping. 


\section{INTRODUCTION}

The level of realism reached today by certain gyrokinetic codes and the computational power that has become available in the last decade have allowed the usage of numerical simulations to model and to some extent also reproduce the behaviour of magnetically confined plasmas, e.g. Ref.1. It is fundamental for such codes to verify that they correctly implement and solve the underlying gyrokinetic model, regardless of the specific numerical algorithm adopted to solve the corresponding equations. The work by Dimits et al. ${ }^{2}$ can be considered among the first systematic benchmark effort of gyrokinetic codes based on a standard test case, the so-called Cyclone Base Case (CBC). Since then, various efforts have already been successfully carried out involving different codes, both in the local (flux tube) limit as well as considering a global representation, i.e. retaining the radial dependence in all equilibrium quantities. ${ }^{3-10}$ With some exceptions, e.g. Refs. $6-8$, these comparisons often use an approximate analytical circular model to describe the plasma equilibrium and adhoc temperature and density profiles. Moreover a simplified adiabatic model for describing the electron dynamics has usually been considered, with the main purpose of reducing the overall required computational cost.

However, because of the continous evolution of the gyrokinetic codes, which are now mostly electromagnetic, multi-species, interfaced to realistic MagnetoHydroDynamic (MHD) equilibria and used to address transport at both ion and electron scales, a more sophisticated series of benchmarks than the standard $\mathrm{CBC}$ case is required to test the codes in all these various regimes. This paper can thus be viewed as a contribution to the current emphasis on validation and verification of fusion-relevant simulations ${ }^{11}$.

We benchmark the three codes GENE, ${ }^{12,13} \mathrm{GKW}^{14,15}$ and $\mathrm{GS} 2^{16}$ in the linear flux tube limit including non-adiabatic electron dynamics and a non-circular plasma shape. We therefore consider here fully gyrokinetic ions and electrons, and we look at geometrical effects by interfacing the codes with realistic numerical MHD equilibria. Carrying out such a benchmark might appear quite straightforward at first glance. Such an effort requires, however, not only to correctly handle the equilibrium provided by an MHD equilibrium solver (we

shall make use of the CHEASE code $^{17}$ for the cases considered here), but also to understand how to cast the results from each code specific coordinate system to a common representation format. This is particularly important when dealing with non-circular plasmas. 
This benchmark effort is in fact a natural extension of what has already been published in Ref. 18, where the five numerical equilibria that are considered here have been introduced. As already pointed out in this original paper, these equilibria are explicitly built to potentially allow both local and global benchmarks and relative comparison between these two representations. In particular, an effort was made to have similar $q$-profiles at all radial positions and not only at a specific location. In this paper any finite machine size effect on instabilities is neglected and left for future study. In carrying out the benchmarks, we proceed in a first stage according to a double blind approach, where an attempt was made with each code results to converge within a certain relative variance level, typically $2-3 \%$, before being confronted with results from the other codes. Then, whenever differences were found, the convergence effort was further pursued. This way of proceeding allowed to reach a $1 \%$ agreement between different codes and also to identify and correct a limited number of code issues, which is exactly the purpose of this benchmark effort.

We put a particular effort in writing this paper to clearly explain all the relevant details, such that anyone can carry out the same series of benchmarks. All the input data, as well as the results, are publicly available online ${ }^{19}$.

The rest of the paper is organized as follows: in Section II we describe the MHD equilibria and the setup of the simulations; in Section III results for the linear stability at both ion and electron scales are compared. In Section IV we benchmark the effect of a finite ballooning angle on the linear stability and finally in Section V the Rosenbluth-Hinton test is carried out looking at the linear dynamics of Zonal Flows (ZFs) and at the properties of the Geodesic Acoustic Mode (GAM). A brief description of the GENE, GKW and GS2 codes is given in Appendix A, B and C, respectively containing a derivation of the relevant geometrical quantities necessary to carry out the benchmarks.

\section{THE BENCHMARK CASES}

For this benchmark we consider five different numerical plasma equilibria obtained using the ideal MHD solver $\mathrm{CHEASE}^{17}$, which provides the axisymmetric equilibrium magnetic field $\mathbf{B}$ with a pressure profile obtained from given density and temperature profiles for the different plasma species. A detailed description of the cases can already be found in 
Ref. 18. For the sake of clarity we briefly present them again here. These equilibria are inspired by the DIII-D shot underlying the Cyclone Base Case ${ }^{2}$ which was a fully shaped plasma corresponding to a Single Null Diverted (SND) configuration. The CBC benchmark, however, just considers circular concentric flux surfaces with only plasma and safety factor profiles taken from the experiment. Among the shapes we consider here, Case I has the most complex geometry and is derived directly from the DIII-D experimental equilibrium i.e. a plasma with up-down asymmetry, positive triangularity $\delta$, elongation $\kappa$ greater than unity and realistic Shafranov shift $\Delta$. The shape complexity is then progressively reduced by removing one shaping effect in each subsequent equilibrium. This is achieved by modifying the Last Closed Flux Surface (LCFS) shape. Therefore Case II is an up-down symmetric triangular plasma, Case III corresponds to an elongated geometry (triangularity $\delta_{\mathrm{LCFS}}=0$ ), Case IV is a circular plasma (elongation $\kappa_{\mathrm{LCFS}}=1$ ), and finally Case $\mathrm{V}$ is a zero $\beta$ plasma with almost concentric flux surfaces (Shafranov shift $\Delta_{\text {LCFS }} \simeq 0$ ). The flux surface contours for the five equilibria are depicted in Figure 1, while the geometrical parameters describing the LCFS are reported in Table I.

Regarding the triangularity of the up-down asymmetric Case I, this shaping parameter has been estimated separately for the upper and lower halves of the corresponding magnetic geometry. This was carried out by considering two separate up-down symmetric equilibria, respectively based on the upper and lower half of the LCFS of Case I, and providing $\delta_{\mathrm{LCFS}}($ upper $)=0.15$ and $\delta_{\mathrm{LCFS}}($ lower $)=0.35$ (see also Table I $)$. Note that the triangularity of Case II is set to $\delta_{\mathrm{LCFS}}=0.15$, i.e. the upper value of Case I.

\begin{tabular}{|c|c|c|c|c|}
\hline Case & $q_{95}$ & $\kappa_{\text {LCFS }}$ & $\delta_{\text {LCFS }}$ & $\Delta_{\text {LCFS }}$ \\
\hline I & 3.92 & 1.68 & $0.15(u) / 0.35(l)$ & -0.0443 \\
\hline II & 3.87 & 1.68 & 0.15 & -0.0531 \\
\hline III & 3.70 & 1.68 & -0.002 & -0.0534 \\
\hline IV & 3.66 & 1.00 & 0.015 & -0.0752 \\
\hline V & 3.72 & 1.00 & 0.0 & -0.0262 \\
\hline
\end{tabular}

Table I. Parameters characterizing the Last Closed Flux Surface of the five equilibrium cases. For the up-down asymmetric geometry of Case I, the values of upper $(u)$ and lower $(l)$ triangularity are provided. 
In order to completely describe the plasma equilibrium it is necessary to specify, in addition to the shape of the LCFS, the safety factor profile as well as the total pressure profile. This is done by choosing similar profiles with respect to the minor radius $r$ for the different equilibria and built such that at the radial position $r / a=0.5$, the local values of safety factor $q_{s}$, magnetic shear $\hat{s}=(r / q)(d q / d r)$ and of temperature and density gradients are as close as possible to the CBC ones $\left(q_{s}=1.4\right.$ and $\hat{s}=0.8, R_{0} / L_{n}=2.22$ and $R_{0} / L_{T}=6.91$ respectively). Throughout all the paper, $r$ indicates the geometric local minor radius of a given flux surface, defined as $r=\left[R_{\max }-R_{\min }\right] / 2, R_{\max }$ and $R_{\min }$ being respectively the maximum and minimum major radius of the flux surface evaluated at the elevation of the magnetic axis. One defines the minor radius $a$ of the LCFS as $a=r(\mathrm{LCFS})$. The geometric center of a given flux surface is defined as $R_{\text {geom }}=\left[R_{\max }+R_{\min }\right] / 2$, such that the major radius of the machine is given by $R_{0}=R_{\text {geom }}$ (LCFS) while the position of the magnetic axis corresponds to $R_{a x i s}=R_{\text {geom }}(0)$. The local aspect ratio is therefore defined as $\epsilon(r)=r / R_{0}$. For all the cases, $a=0.6 \mathrm{~m}$ and $R_{0}=1.68 \mathrm{~m}$ have been taken, corresponding to an inverse aspect ratio $a / R_{0}=0.36$, and the flux tube considered in the gyrokinetic simulations has been centered at $r / a=0.5$, such that $\epsilon=0.18$. For all the simulations, a
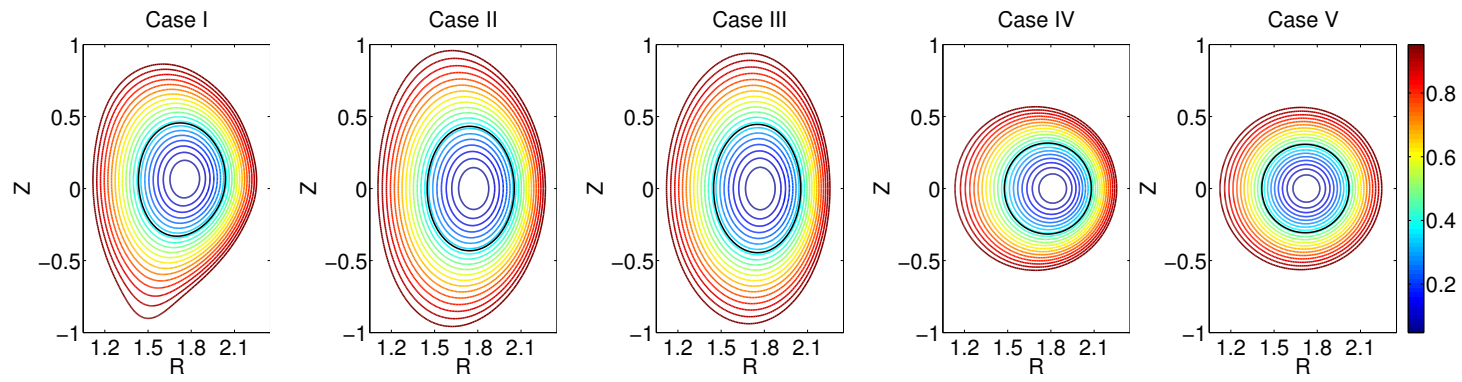

Figure 1. MHD equilibrium Cases I to V. Shown are constant contours of the poloidal magnetic flux function $\psi / \psi(\mathrm{LCFS})$. The contour of the flux surface at $r / a=0.5$ considered for the local simulations is depicted in black (Color online).

deuterium plasma is considered (assuming real mass ratio $m_{D} / m_{e}=3670, m_{D}$ and $m_{e}$ being respectively deuterium and electron masses), and as the benchmark is carried out in the flux tube limit, only the values of normalized inverse temperature and density gradient lengths $R_{0} / L_{T, n}=R_{0} d \log (T, n) / d r$ at the position of interest are required. As already mentioned, these values are set to $R_{0} / L_{T}=6.91$ and $R_{0} / L_{n}=2.22$ for all the five cases. The same temperature is assumed for ions and electrons $\left(\tau=T_{e} / T_{i}=1\right)$. Collisions are neglected 
and no background flows are considered. Note that the inverse gradient lengths $R_{0} / L_{T, n}$ are evaluated as derivatives of the profiles with respect to the geometric minor radius $r$. The true normalized gradients driving the instabilities at a given position on a magnetic surface are however given by $R_{0}|\nabla \log (T, n)|=R_{0} d \log (T, n) / d r|\nabla r|$, where the geometrical factor $|\nabla r|$ is in general different from unity and not constant on a magnetic surface in a shaped plasma. One may thus estimate on a given flux surface an effective flux-surface-averaged gradient given by $\left\langle R_{0}|\nabla \log (T, n)|\right\rangle=R_{0} / L_{T, n}\langle|\nabla r|\rangle$. Here $\langle\mathcal{A}\rangle$ indicates the flux surface average of a quantity $\mathcal{A}$ and is defined by

$$
\langle\mathcal{A}\rangle=\lim _{\Delta \psi \rightarrow 0} \frac{1}{\Delta V} \int_{\Delta V} \mathcal{A} d^{3} x
$$

where $\Delta V(\psi)$ is the volume between the flux surface $\psi=$ const and $\psi+\Delta \psi=$ const. The corresponding values of $\langle|\nabla r|\rangle$ evaluated at $r / a=0.5$ are listed in Table II and shown as a function of elongation in Figure 2. Note that in all the graphs comparing results pertaining to different geometries, the same color coding has been used to help the reader (color online): red for Case I, blue for Case II, black for Case III, green for Case IV and magenta for Case V. The nice alignment of the data in this plot already hints towards elongation being the most important shaping parameter considered here. Note that for all the Cases with elongation, the mid-radius values differ while the edge elongations are kept constant $\kappa_{L C F S}=1.68$ (see Tables I and III). Elongation penetrates differently with different edge triangularities, as noted in Ref. 20, and a lower $\delta$ leads to a larger $\kappa$ in the core (Case III).

\begin{tabular}{|c|c|c|c|c|c|}
\hline Case & I & II & III & IV & V \\
\hline$\langle|\nabla r|\rangle$ & 0.907 & 0.871 & 0.864 & 1.040 & 1.003 \\
\hline$\left\langle R_{0}|\nabla \log n|\right\rangle$ & 2.014 & 1.933 & 1.918 & 2.308 & 2.226 \\
\hline$\left\langle R_{0}|\nabla \log T|\right\rangle$ & 6.269 & 6.017 & 5.969 & 7.184 & 6.929 \\
\hline
\end{tabular}

Table II. Values of flux surface averaged $\langle|\nabla r|\rangle$ and the corresponding values of effective flux-surface averaged temperature and density gradient lengths for the five Cases $\left(R_{0}\langle|\nabla \log (T, n)|\rangle=R_{0} / L_{n, T}\langle|\nabla r|\rangle\right)$.

Of particular interest in this benchmark is the possibility to study the effect of the usual shaping parameters on plasma behaviour by simply comparing the five Cases. We note that when moving from one case to another, although one specific edge shaping parameter is 


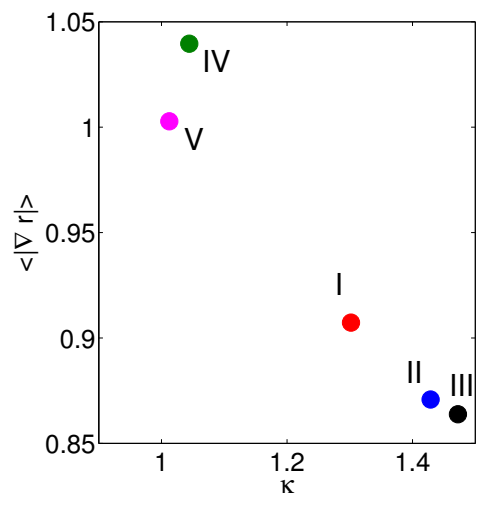

Figure 2. Flux surface averaged $\langle|\nabla r|\rangle$ evaluated at $r=0.5$ versus elongation $\kappa$. Numbers identify the corresponding case.

sensibly varied intentionally, all the other parameters change to some degree as well.

In order to facilitate the understanding of the effect of the various shaping parameters, an interface with Miller's equilibrium ${ }^{21}$ will be exploited in a few cases (results shown in Figs. 6 and 15) using the GENE code. The Miller parametrization considered in GENE parametrizes in a poloidal plane $\varphi=$ const the contour of a given magnetic surface with geometric minor radius $r=$ const by a poloidal angle $\theta$ (in general different from the geometric angle) and is given in cylindrical coordinates $(R, Z, \varphi)$ by $^{22}$

$$
\begin{aligned}
& R(r, \theta)=R_{\text {geom }}(r)+r \cos \{\theta+\arcsin [\delta(r) \sin \theta]\} \\
& Z(r, \theta)=Z_{\text {geom }}(r)+\kappa(r) r \sin [\theta+\zeta(r) \sin (2 \theta)],
\end{aligned}
$$

where the elongation $\kappa$, triangularity $\delta$ and squareness $\zeta$ have been introduced. $Z_{\text {axis }}$

\begin{tabular}{|c|c|c|c|c|c|c|c|c|c|c|c|}
\hline Case & $q_{s}$ & $s$ & $\kappa$ & $\delta$ & $\zeta$ & $\Delta$ & $\alpha_{\mathrm{MHD}}$ & $d R_{\text {geom }} / d r$ & $d \kappa / d r$ & $d \delta / d r$ & $d \zeta / d r$ \\
\hline I & 1.382 & 0.804 & 1.3015 & 0.0812 & $9.0110^{-4}$ & -0.0127 & 0.515 & -0.1383 & -0.0765 & 0.1065 & -0.02 \\
\hline II & 1.382 & 0.778 & 1.4287 & 0.0260 & $5.2210^{-4}$ & -0.0132 & 0.5338 & -0.1488 & -0.0766 & 0.0437 & -0.054 \\
\hline III & 1.389 & 0.751 & 1.4723 & -0.0070 & $2.8310^{-3}$ & -0.0139 & 0.5425 & -0.1569 & -0.0728 & -0.0140 & 0.003 \\
\hline IV & 1.450 & 0.764 & 1.0443 & 0.0065 & $5.1310^{-4}$ & -0.0206 & 0.5552 & -0.2269 & -0.0239 & 0.0001 & 0.01 \\
\hline V & 1.427 & 0.847 & 1.0124 & 0.0014 & $1.0410^{-4}$ & -0.0045 & 0.0 & -0.0587 & -0.0036 & 0.0148 & 0.002 \\
\hline
\end{tabular}

Table III. Parameters characterizing the five flux surfaces of interest computed according to Eqs.(2) and (3). 

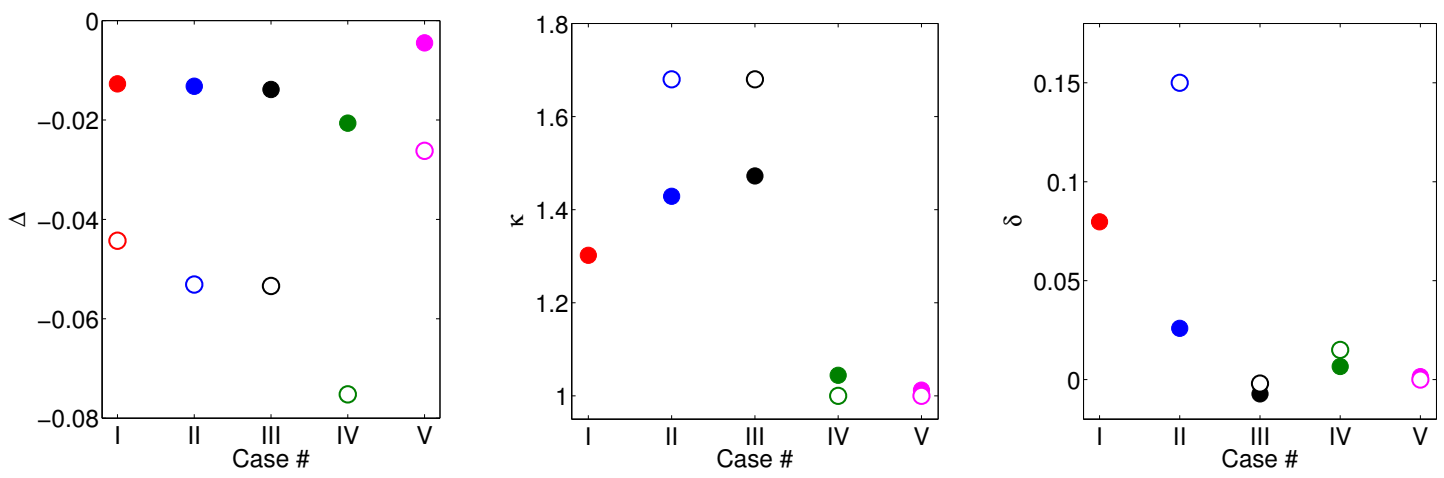

Figure 3. Values of the usual shaping parameters: (a) Shafranov shift $\Delta,(b)$ elongation $\kappa$ and (c) triangularity $\delta$ evaluated at the position $r / a=0.5$ for the five test cases. The same quantities measured at the LCFS are shown with empty symbols. The values of $\kappa$ and $\delta$ are computed according to the Miller parametrization given in Eqs.(2) and (3), and therefore shown only for the plasma boundary of the up-down symmetric geometries (cases II to V), whereas for all equilibria the Shafranov shift $\Delta$ is evaluated as $\Delta=\left[R_{\text {geom }}-R_{\text {axis }}\right] / R_{\text {axis }}$.

indicates the elevation of the magnetic axis with respect to the equatorial mid-plane. In order to evaluate these parameters, the global CHEASE equilibrium is fitted according to Eqs. (2) and (3) and the radial derivatives of the shaping parameters $\left[\kappa^{\prime}(r), \delta^{\prime}(r), \zeta^{\prime}(r)\right]$, also required for implementing the Miller equilibrium in the gyrokinetic equations, are evaluated in the neighborhood of the flux surface of interest. The parametrization given by Eqs. (2) and (3) does not require to specify the value of the Shafranov shift, instead it requires the value of $d R_{\text {geom }} / d r$ to build all the geometrical quantities required to solve the gyrokinetic equations. The actual parameters characterizing the flux surface $r / a=0.5$ are listed in Table III for the five equilibria considered. For completeness the values of the Shafranov Shift $\Delta$, defined as $\left[R_{\text {geom }}(r)-R_{\text {axis }}\right] / R_{\text {axis }}$, and $\alpha_{\mathrm{MHD}}=-q_{s}^{2} R_{0}(d \beta / d r)$ where $\beta=2 \mu_{0} p / B_{\text {mag }}^{2}, p$ and $B_{\text {mag }}^{2} / 2 \mu_{0}$ being respectively the local thermal pressure provided by the MHD equilibrium and the magnetic pressure estimated with the magnetic field $B_{\text {mag }}$ on axis, are tabulated as well. As depicted in Figure 3 and quantified in Table III, the different shaping parameters have different radial penetration depths. In particular it is to be noted for Case II, with finite triangularity $\delta_{\mathrm{LCFS}}=0.15$ at the LCFS, that the remaining triangularity at $r / a=0.5$ is reduced by more than $80 \%$ to $\delta=0.026$, illustrating the weak penetration depth of this shaping parameter. We also remark that the higher triangularity 
that characterises Case I is due to an effectively higher $\delta_{\mathrm{LCFS}}$ of the fully shaped plasma compared to Case II. Elongation however, which remains finite down to the magnetic axis and as noted above is favored by small $\delta$, is for example reduced only by $\approx 10 \%$ in Case III, going from $\kappa_{L C F S}=1.68$ to $\kappa=1.47$ at $r / a=0.5$.

\section{LINEAR $k_{\theta}$-SPECTRA}

First, a linear electrostatic benchmark is performed, considering instability spectra with wave-numbers up to the electron Larmor radius scales. All the five cases described in Sec. II are characterized by a mixed Ion Temperature Gradient (ITG) - Trapped Electron Modes (TEM) regime at the ion Larmor radius scale, as already shown in Ref. 18, while Electron Temperature Gradient (ETG) driven modes dominate the spectra at electron Larmor radius scales. The finite pressure gradient contribution is kept in computing the curvature drift $\mathbf{v}_{c}$ for a given species $j$ :

$$
\mathbf{v}_{c}=\frac{v_{\|}^{2}}{\Omega_{j}}\left(\nabla \times \frac{\mathbf{B}}{B_{\perp}}\right)=\frac{v_{\|}^{2}}{\Omega_{j}} \frac{\mathbf{B}}{B} \times\left(\nabla \ln B+\frac{\beta}{2} \nabla \ln p\right)
$$

having made use of the MHD equilibrium force balance $\nabla p=\mathbf{j} \times \mathbf{B}$ and Ampére's law $\nabla \times \mathbf{B}=\mu_{0} \mathbf{j}, \mathbf{j}$ standing for the plasma current and $\Omega_{j}=q_{j} B / m_{j}$ the cyclotron frequency for species $j$ with mass $m_{j}$ and electric charge $q_{j}$. The value of $\beta$ is computed consistently with the CHEASE equilibrium. The effect of the pressure gradient is small but not negligible, especially at the ITG to TEM transition, which is shifted to lower wave-numbers when this pressure term is accounted for.

\section{A. The GENE code}

At ion scales, the GENE code has been run in its eigenvalue (spectral) version ${ }^{23-25}$ in order to recover not only the most unstable mode but the subdominant branches of the dispersion relation as well. At ETG scales, because of the absence of strong subdominant modes, the initial value (time evolution) approach was used for being significantly more effective than the eigenvalue procedure in determining the growth rates of the most unstable modes. The GENE code employs a field-aligned coordinate system $(x, y, z)$ to represent the fluctuation 
fields in configuration space. Here $x$ defines the radial direction, $y$ the binormal and $z$ parametrizes a field line, thus the latter is usually referred to as the "parallel" direction. Parallel velocity $v_{\|}$and magnetic moment $\mu$ are the velocity space variables. For a more detailed description, the reader is referred to Appendix A and Refs. 12 and 26. In the fluxtube limit, both the radial $x$ and binormal $y$ directions are Fourier transformed. For the considered axisymmetric system, linear modes have a fixed $k_{y}$ Fourier mode (axisymmetry corresponds to invariance in the $y$ direction), which is related to a toroidal mode number $n$ according to Eq. A5. All the simulations have been carried out using $n_{k_{x}}=32$ radial modes connected because of the parallel boundary condition, while $n_{z}=64$ points have been used to discretize the "parallel" direction z. For the velocity space, unless specified differently, a uniform grid composed of $n_{v_{\|}}=128$ points between $0<v_{\|}<4.24 v_{\mathrm{j}}$ was used to discretize $v_{\|}$direction, while $n_{\mu}=32$ Gauss-Laguerre integration points between $0<\mu<9 T_{j} / B_{0}$ were used for the $\mu$ direction. Here $v_{\mathrm{j}}=\sqrt{T_{j} / m_{j}}$ stands for the thermal velocity of species $j$ (we note that the different codes use different normalization rules, the reader is referred to Appendixes A 3, B 2 and C 2 for more details regarding GENE, GKW and GS2 codes respectively).

\section{B. The GKW code}

The GKW code also considers a field-aligned coordinate system noted $(r, \zeta, s)$ built from the Hamada coordinates. Here $r$ is the radial direction, $\zeta$ the binormal and $s$ (one of the Hamada coordinates) is referred to as the "parallel" direction; as for the GENE code, parallel velocity $v_{\|}$and magnetic moment $\mu$ are used for discretizing the velocity space. The GKW coordinate system is briefly presented in Appendix B, a detailed description is given in Ref. 14. Again a Fourier decomposition is used for the flux-tube representation in both $r$ and $\zeta$ directions. The simulations have been carried out considering $n_{k_{r}}=25$ radial modes $k_{r}$ connected via the parallel boundary condition, while $n_{s}=35$ points were used to discretize the $s$ direction. Uniform grids in both $v_{\|}$and $\mu$, with the same upper limits as considered for GENE, have been adopted for the velocity space and discretized using $n_{v_{\|}} \times n_{\mu}=128 \times 8$ points respectively. 


\section{The GS2 code}

The GS2 coordinate system $(X, Y, \theta)$ and its Fourier representation are directly related to the ballooning representation of a fluctuating field (see Appendix $\mathrm{C}$ and Ref. 16 for the details). All the simulations shown here have been performed considering $n_{k_{X}}=15$ connected radial modes, while a parallel resolution of $n_{\theta}=32$ has been used to discretize a magnetic field line along one poloidal turn. Differently from GENE and GKW, in the GS2 code energy $\mathcal{E}$ and pitch angle $\lambda=v_{\perp}^{2} /\left(v^{2} B_{0}\right)$ are used as velocity space variables to represent the distribution function, while the integration is carried out according to a Gauss-Legendre distribution of points. For these simulations $n_{\lambda}=24$ points are used to discretize the $\lambda$ direction while $n_{\mathcal{E}}=18$ for $\mathcal{E}$. The maximum value of the $\mathcal{E}$-grid is set to $11.3 m_{j} v_{j}^{2} / 2$.

\section{Results}

We point out that the simulations performed with the GENE code have been carried out with an higher resolution in both the radial and "parallel" directions with respect to GKW and GS2 with the aim of providing reference results well converged in all directions. We nevertheless remark that using a lower resolution e.g. $n_{k_{x}} \times n_{z} \times n_{v_{\|}} \times n_{\mu}=32 \times 32 \times 64 \times 16$ in GENE is sufficient to provide growth rates and frequencies converged within $5 \%$ for all the microturbulence regimes and plasma shapes being considered here. We also note that the efficient parallelization scheme adopted in GENE allows to carry out the most resolved runs without a significant increase of the computational cost. A similar strategy was used for setting the discretization of the velocity space, which as will be shown turns out to be crucial, especially as one needs to correctly resolve the trapped-passing particle boundary. The choice of $\left(v_{\|}, \mu\right)$ naturally requires a higher number of points to resolve this boundary compared to a discretization along $(\mathcal{E}, \lambda)$, which explains the difference between the GENE and GKW setups with respect to GS2. The trapped-passing boundary is indeed aligned to a Cartesian $(\mathcal{E}, \lambda)$ grid while it is diagonal to a $\left(v_{\|}, \mu\right)$ grid.

One of the main difficulties arising when comparing different codes is the dependence of the corresponding results on code-specific coordinate systems. This is particularly critical when considering plasmas with non-circular shapes, as differences between various coordinate systems become significant. We recall that for linear modes in an axisymmetric systems, $n$ is 
an exact mode number, while this is not the case for poloidal mode numbers $m$. Nonetheless, as the fluctuations are field aligned, poloidal wave numbers can be estimated as $k_{\theta} \sim m / r$ with $m \sim n q_{s}$. Therefore we plot the real frequencies $\omega_{r}$ and the growth rates $\gamma$ of the modes (in units of $v_{i} / R_{0}$ ) with respect to the effective poloidal mode number estimate $k_{\theta}=n q_{s} / r_{0}$ normalized to the ion Larmor radius $\rho_{i}=v_{i} / \Omega_{i}$. This quantity can be evaluated from each code's specific representation (see Appendix A, B and C for details respectively to codes GENE, GKW and GS2):

$$
\begin{array}{ll}
n=k_{y} C_{y} & \text { for GENE } \\
n=\frac{k_{\zeta}}{2 \pi} & \text { for GKW } \\
n=k_{Y} \frac{1}{B_{a}} \frac{d \psi}{d r} & \text { for GS2 }
\end{array}
$$

Throughout the paper we conform to GENE conventions on the sign of the real frequency of the mode: a positive value indicates a propagation in ion diamagnetic drift direction while a negative value corresponds to propagation in the electron diamagnetic direction.

The results obtained for the $k_{\theta}$-spectra using the three codes are shown in Figure 4 at the ion scales $\left(k_{\theta} \rho_{i} \sim 1\right)$, while in Figure 5 the linear spectra at the electron scales $\left(k_{\theta} \rho_{e} \sim 1\right)$ are compared for Case I. All results shown here consider zero ballooning angle $\chi_{0}=0$, where $\chi$ is the straight field-line poloidal angle as defined in Eq. (A2). With the previously specified setup, GENE, GKW and GS2 agree between each other within 3\% on both real frequency $\omega_{r}$ and growth rate $\gamma$ of the most unstable modes, at all scales and independently from shaping. Separate convergence studies have been performed at the different scales and the difference between the codes was further reduced to $1 \%$ when higher resolutions, similar to the GENE ones, were considered by the different codes. We note that the transition from ITG to TEM cannot be taken as a practical benchmark point as its position is very sensitive to the resolution used and a convergence study is particularly expensive.

As already discussed in Sec. II, when going from one equilibrium case to the other, all the parameters characterizing the actual local flux surface geometry are changed. The interface to the Miller equilibrium given in Eqs. (2) and (3) has therefore been used to study the effect of each parameter separately. Starting from the representation of the flux surface of Case V, one geometrical parameter is changed at the time, until reaching the values characteristic of Case I. The results, obtained with GENE for $k_{y} \rho_{i}=0.3$, are presented in Figure 6, where the labels indicate the particular parameter being changed (either $q_{s}, \hat{s}, \epsilon, \kappa, \delta$ or $d R / d r$ ). 

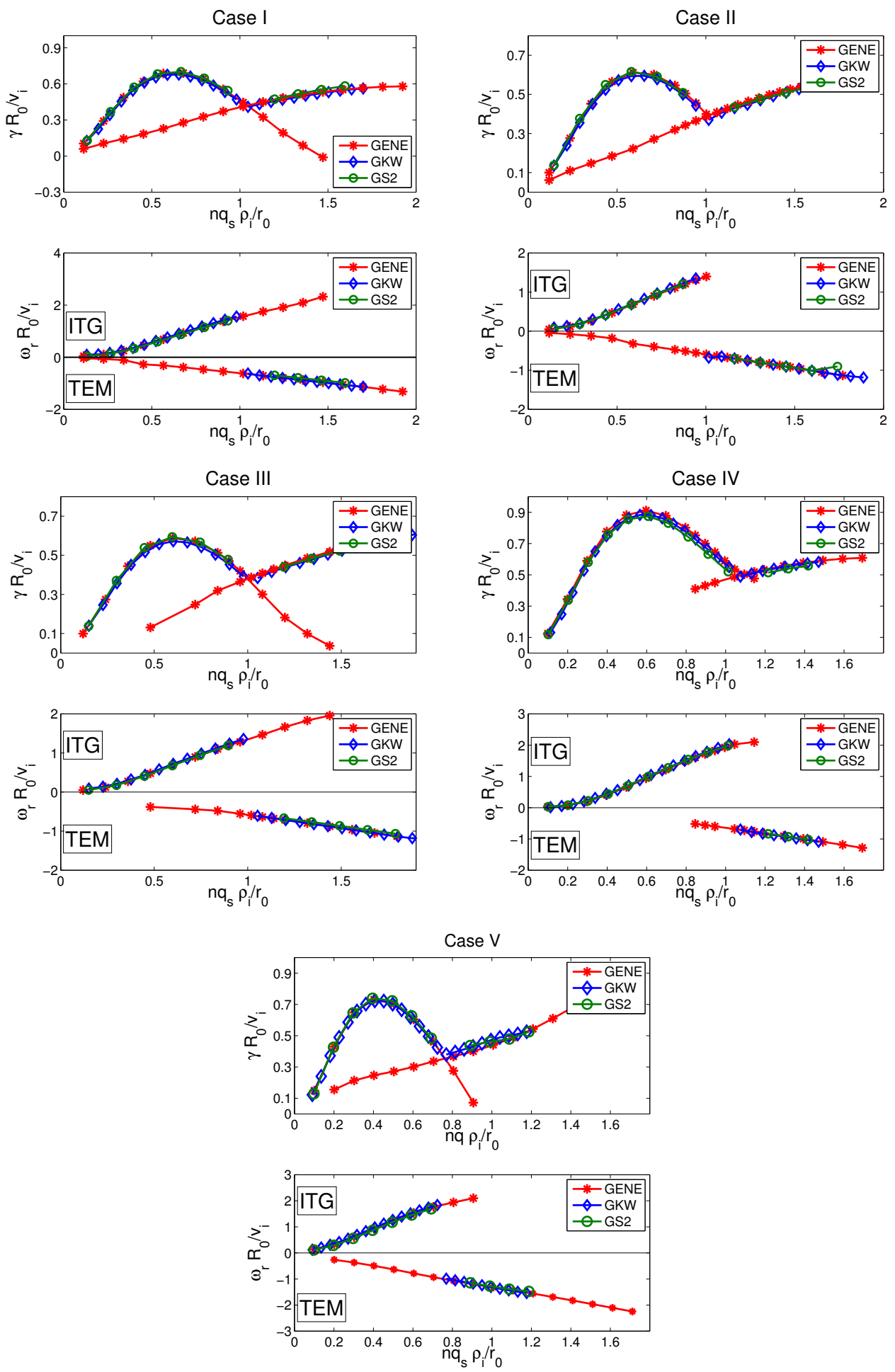

Figure 4. (Color online) Real frequency $\omega_{r}$ and growth rates $\gamma$ normalized to $R_{0} / v_{i}$ as a function of the effective poloidal mode number $k_{\theta} \rho_{i}=n q_{s} \rho_{i} / r_{0}$ for the five CHEASE equilibrium test cases. Shown are the results obtained with the GENE (red stars), GKW (blue diamonds) and GS2 (green circles) codes. Sub-dominant modes are only provided for GENE results. 

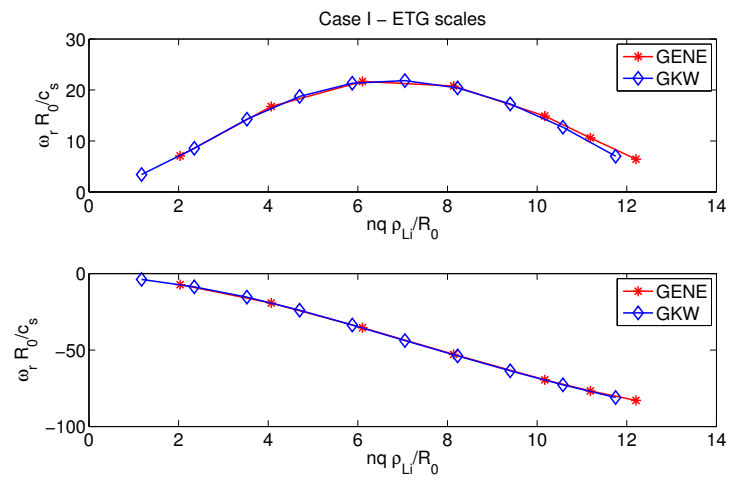

Figure 5. Benchmark at electron scales for Case I. Results from GENE (red stars), GKW (blue diamonds) and GS2 (green circles).
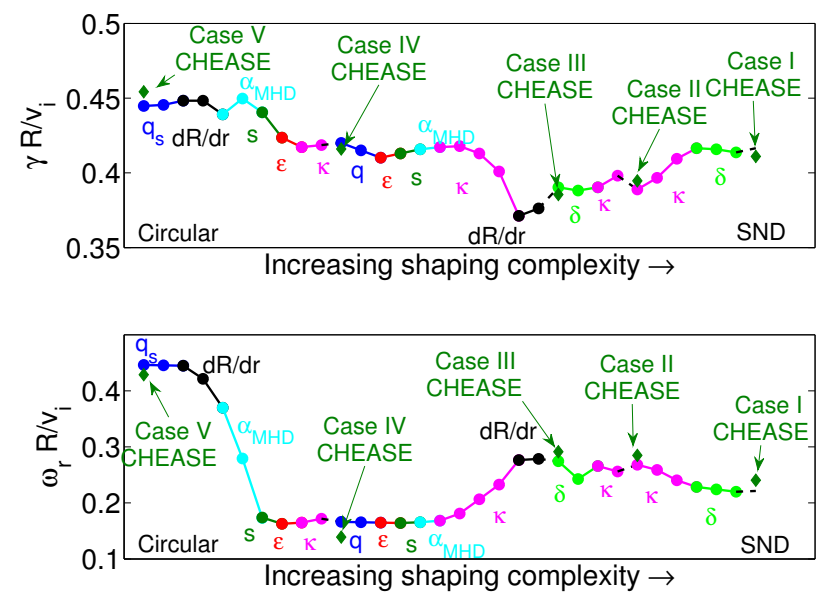

Figure 6. (Color online) Variation of growth rate $\gamma$ and real frequency $\omega_{r}$ for fixed $k_{y} \rho_{i}=0.3$ (according to GENE convention) continuously increasing shaping complexity from Case V (circular, $\beta=0$ ) to I (SND, $\beta \neq 0$ ) making use of the Miller equilibrium representation. Labels indicate the parameter being changed at each step. Results obtained for the five cases with the MHD equilibrium are shown for comparison and labeled "CHEASE" (green diamonds). All results have been obtained with the GENE code.

Dashed lines are used for illustrating the combined variation related to parameters inducing only smaller effects. We note that having performed this scan at fixed $k_{y} \rho_{i}$, the equivalent $k_{\theta} \rho_{i}$ as defined in Eqs. (5-7) is not constant because of the variation in both $n$ and $q_{s}\left(k_{\theta} \rho_{i}\right.$ varies between 0.296 and 0.451$)$.

From Figure 6 it appears, as expected, that any variation of the parameters used to describe 
the plasma equilibrium contributes to a change in the linear spectra. For each change of equilibrium between Cases V and III it is possible to identify one parameter which leads to the most significant variation: $\alpha_{\text {MHD }}$ going from Case V to IV (responsible for the Shafranov shift), elongation $\kappa$ going from Case IV to III. Note that going from Case III to II, very little variation arises from the change in triangularity, while the effect of the variation of $\kappa$ is comparable to the results of changing all the other parameters. Going from Case II to I, where despite the up-down asymmetry the Miller parametrization still is a good approximation, elongation appears once again as the main reason for change in growth rate and frequency of the mode. Triangularity $\delta$, despite the significant relative variation at the considered reference surface (see table III), has only a little effect.

Considering the relative variation of the growth rate for a given relative variation of the

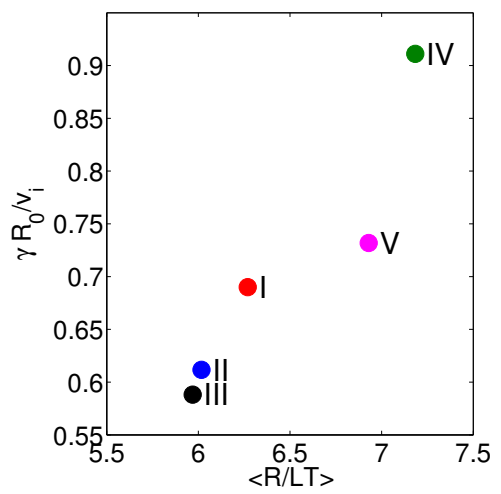

Figure 7. Maximum linear growth rate in ITG regime for all five Cases versus the flux surface averaged temperature gradient length. Values in units of $R_{0} / v_{i}$. Numbers identify the corresponding case. Results from the GENE code.

various shaping parameters considered here, elongation appears the most important, and the change in linear stability from Case IV to I can be explained mainly by a variation in $\kappa$. As an example, in Figure 7 we plot $\gamma$ of the most unstable mode in the ITG regime for the five test Cases versus the corresponding effective temperature gradient (which is determined mainly by elongation, as shown in Fig. 2). A trend is clearly recovered; we plot also the result obtained for Case $\mathrm{V}$ which appears not as much aligned as the other points. This happens because of the strong effect exerted by $\alpha_{\mathrm{MHD}}=0$ and $d R_{\text {geom }} / d r$ in going from Case IV to V. 


\section{BALLOONING ANGLE SCAN}

\section{A. Mode frequency and growth rate}

The effect of a finite ballooning angle $\chi_{0}$ is usually neglected in linear studies and benchmarks, which are normally carried out under the assumption of zero $\chi_{0}$. However, linearly ${ }^{27-29}$ and non linearly correctly taking into account ballooning angle is crucial to accurately predict the transport level, thus the reason for benchmarking this effect. We recall that the ballooning angle is the angle at which turbulent eddies point radially.

A finite ballooning angle can be introduced in the Fourier representation of all the codes used. For a given $k_{y}$ (resp. $k_{\zeta}, k_{Y}$ ) linear mode, GENE (resp. GKW, GS2) code couples the radial Fourier modes $k_{x}=\delta k_{x}+p \Delta k_{x}\left(\operatorname{resp} . k_{r}=\delta k_{r}+p \Delta k_{r}, k_{X}=\delta k_{X}+p \Delta k_{X}\right)$, $p \in \mathbb{Z}$, where $\delta k_{x}, \delta k_{r}$ and $\delta k_{X}$ are related to the straight field poloidal ballooning angle $\chi_{0}$ by the relations (see Appendix A, B and C for details on GENE, GKW and GS2 codes respectively):

$$
\begin{array}{ll}
\chi_{0}=-\frac{\delta k_{x}}{k_{y} \hat{s}} & \text { for GENE } \\
\chi_{0}=-2 \pi \frac{\delta k_{r}}{k_{\zeta} d q / d r} & \text { for GKW } \\
\chi_{0}=-\frac{\delta k_{X}}{k_{Y} \hat{s}} & \text { for GS2 }
\end{array}
$$

For each of the five test cases, a scan of $\chi_{0}$ has been carried out at given toroidal mode number, $\left.k_{\theta}\right|_{\text {GKW }} \rho_{i}=0.2$ according to GKW definitions, the corresponding values of $n q_{s} \rho_{i} / r_{0}$ are listed in Tab. IV. For the sake of completeness the input values of $\left.k_{y} \rho_{i}\right|_{\text {GENE }}$ and $\left.k_{Y} \rho_{i}\right|_{\text {GS2 }}$ used for the equivalent GENE and GS2 runs are reported as well. The resolution $n_{k_{x}} \times n_{z} \times n_{v_{\|}} \times n_{\mu}=32 \times 64 \times 128 \times 32$ has been used for the simulations performed with the GENE code, while GKW runs have been carried out considering $n_{k_{r}} \times n_{s} \times n_{v_{\|}} \times n_{\mu}=$ $27 \times 35 \times 128 \times 32$. GS2 runs have been performed using $n_{k_{X}} \times n_{\theta} \times n_{\lambda} \times n_{\mathcal{E}}=63 \times 48 \times 24 \times 18$ grid points. The results obtained are shown in Figure 8, where growth rates and frequencies are plotted as a function of $\chi_{0}$ for the five geometries.

We note that increasing the ballooning angle the ITG mode, which is the most unstable one associated to $\chi_{0}=0$, is first stabilized. Then at larger values of $\chi_{0}\left(\chi_{0} \sim \pm 0.3\right)$ a transition to TEM is found for all Cases except Case IV, where the mode is stable. With the GENE code, used in its initial value mode of operation, we resolved the mode transition 


\begin{tabular}{|c|c|c|c|c|c|}
\hline Case & I & II & III & IV & V \\
\hline$n q_{s} \rho_{i} / r_{0}$ & 0.180 & 0.222 & 0.308 & 0.312 & 0.276 \\
\hline$\left.k_{y} \rho_{i}\right|_{\text {GENE }}$ & 0.231 & 0.246 & 0.251 & 0.227 & 0.179 \\
\hline$\left.k_{\theta} \rho_{i}\right|_{\text {GKW }}$ & 0.2 & 0.2 & 0.2 & 0.2 & 0.2 \\
\hline$\left.k_{Y} \rho_{i}\right|_{\text {GS2 }}$ & 0.197 & 0.196 & 0.201 & 0.223 & 0.183 \\
\hline
\end{tabular}

Table IV. Equivalent poloidal mode number $n q_{s} \rho_{i} / a$ used for the finite ballooning angle scan in the five test Cases. The corresponding GENE, GKW and GS2 input binormal wave vectors $\left.k_{y} \rho_{i}\right|_{\text {GENE, }}$ $\left.k_{\theta} \rho_{i}\right|_{\mathrm{GKW}}$ and $\left.k_{Y} \rho_{i}\right|_{\mathrm{GS} 2}$, are tabulated as well.

systematically for all geometries. As can be seen from Fig. 8 a good agreement is again recovered between the codes, within $3 \%$ on both frequency and growth rate. As for the $k_{\theta}$-spectra, the transition point is strongly dependent on the resolution adopted and cannot be taken as an exact benchmark point (a convergence study being too costly).

We also remark that for this particular value of $n q_{s} \rho_{i} / r_{0}$ in spite of the up-down edge asymmetry, the most unstable modes for Case I are indeed associated to an almost zero ballooning angle (the difference between growth rates at positive and negative $\chi_{0}$ is of the order of $\simeq 2 \%$, with more unstable modes at positive angles). This is because the flux surface of interest is far inside the plasma cross section, such that the effective up-down asymmetry is very weak.

\section{B. Mode structure}

Finally, we benchmark the mode structure of the electrostatic potential $\phi$ associated to some of the modes for which frequency and growth rate have been computed in the previous section. In order to compare the results from different codes, amplitudes and phases of the fields must be appropriately renormalized; we therefore plot $\phi(\chi)$ renormalized such that $\Re\{\phi(\chi=0)\}=1$ and $\Im\{\phi(\chi=0)\}=0$.

Benchmarking the ballooning structure turns out to be very challenging, more than the growth rate and frequency of the mode. In particular, we note that when growth rate and frequency of the mode are converged within few percents $(\sim 5 \%)$, then the most ballooned part of the mode, i.e. $-\pi \leq \chi \leq \pi$ is also converged and a good agreement between the 

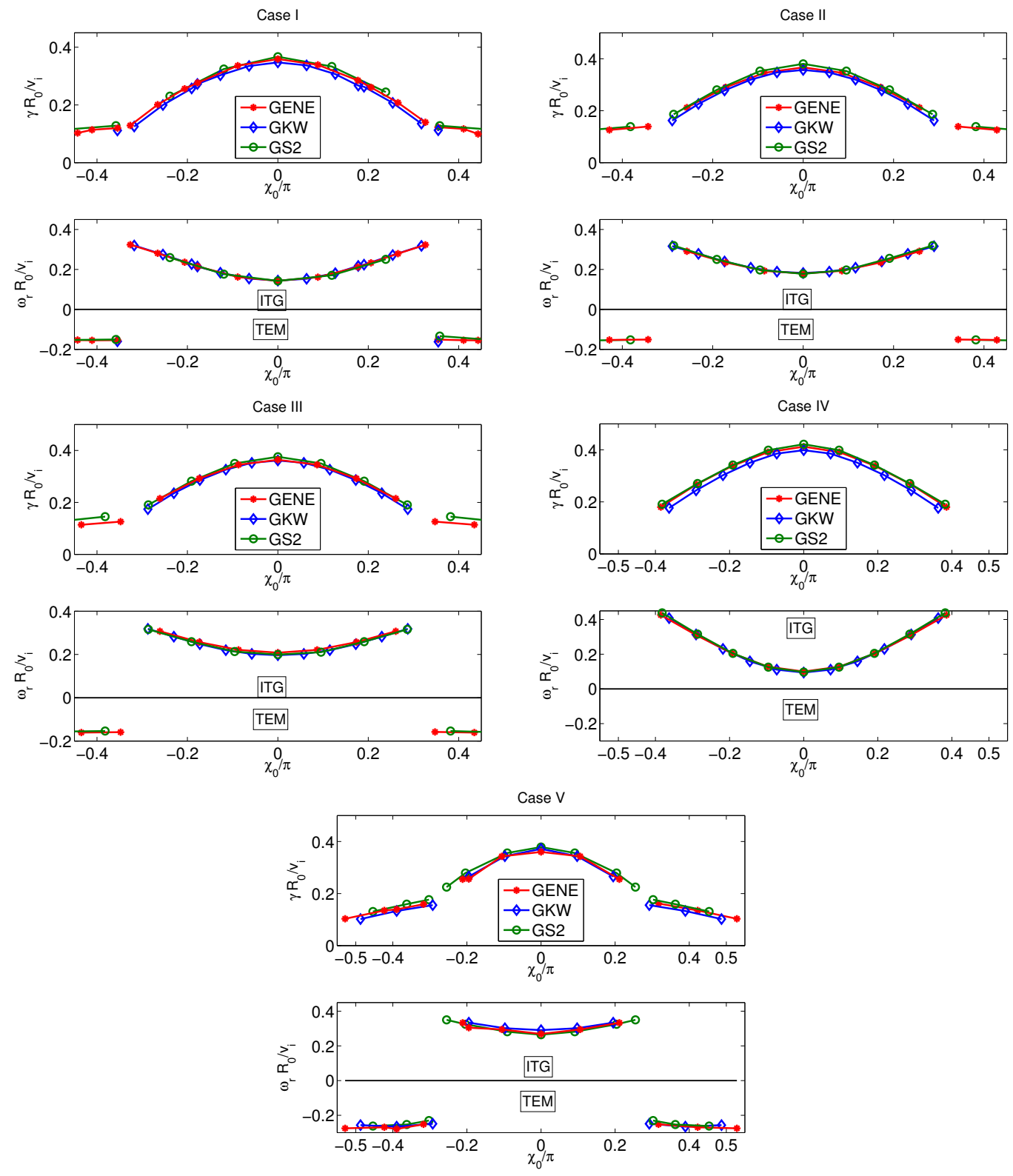

Figure 8. Ballooning angle scan for the five test cases. From Case I to V growth rates and frequency in units of $v_{i} / R_{0}$ are shown for the GENE code (red) code, GKW (blue) and GS2 (green).

codes is recovered. An example is given in Figure 9, where the eigenfunction of the mode $n q_{s} \rho_{i} / r_{0}=0.276$, computed considering the magnetic geometry of Case $\mathrm{V}$ and $\chi_{0}=0$, is compared. The results of Fig. 9 have been obtained using the same resolutions as adopted for computing real frequency and growth rate of the mode, and one observes differences in the tails of the eigenfunction, for $\chi>2 \pi$. We nevertheless remark that even if the local differences can be up to $30 \%$, they remain small compared to the maximum amplitude of 


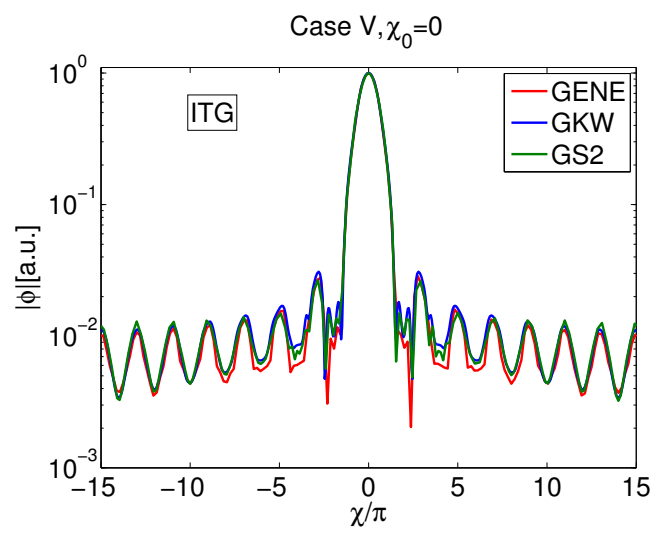

Figure 9. (Color online) Ballooning representation of the electrostatic potential $\phi$ computed with GENE (red), GKW (blue) and GS2 (green) for the mode $n q_{s} \rho_{i} / r_{0}=0.276$ and $\chi_{0}=0$. Magnetic geometry of Case V.

the mode.

In order to converge the mode structure and have the same good match between codes over a wider range of $\chi$ values, the resolution has to be significantly increased. Besides a sufficiently large number of connections along the field line, corresponding e.g. to the number of $k_{x}$ modes in GENE, the velocity space resolution turns out to be crucial for recovering a good agreement over the complete ballooning structure. This is especially true for the TEM modes, for the same reasons as described in Sec. III. This turns out to be a challenging and computationally significant effort, therefore it has been limited to GENE and GKW codes only. No particular reason prevents from doing the same also with GS2. Also, this benchmark is carried out considering only Case V and I, viz. the circular and the fully shaped geometry, while values of $\chi_{0}$ have been selected such that both ITG and TEM regime are studied.

The results obtained are shown in Figure 10 for Case V and Figure 11 for Case I. All these simulations have been performed using $64 n_{k_{x}}$ modes in GENE and $64 n_{k_{r}}$ in GKW, while 70 points have been used to discretize along the magnetic field line. The runs associated to ITG modes have been carried out discretizing the phase space with $n_{v_{\|}} \times n_{\mu}=128 \times 32$ points in both codes, while for TEM modes the velocity space resolution has been further increased to $192 \times 48$ in GENE and $256 \times 64$ in GKW. With these grids the frequency obtained from the two codes agree within less than 1\%. The different number of grid points required for velocity space is explained by their different distribution. We note that the two codes adopt 
different boundary conditions at the beginning and end of a magnetic field line: in GENE, the default setting is assuming zero perturbation at the domain boundaries (other options are available). In GKW a zero derivative condition is applied (see Refs. 14 and 26 for more details on the actual implementation). We have verified by further increasing the number of connected radial modes that the boundary condition is not affecting the results. We also note that reducing parallel dissipation in GKW appears to further improve the agreement. However, this requires to set a smaller time step increasing the cost of the runs, so we have carried out all the simulations setting the dissipation to 0.02 without further pushing the convergence.
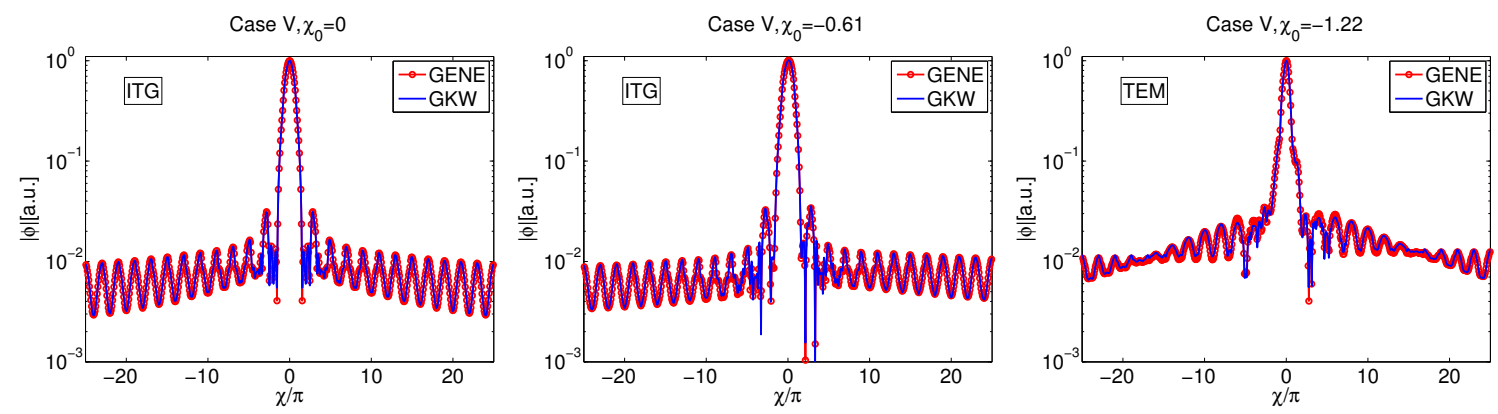

Figure 10. (Color online) Ballooning representation of the electrostatic potential $\phi$ computed with GENE (red) and GKW (blue) for three different values of ballooning angle $\chi_{0}$. Shown are the results obtained for $(a)$ zero ballooning angle, $(b)$ finite $\chi_{0}$ in the ITG regime and $(c)$ finite $\chi_{0}$ for TEM. The value of $\chi_{0}$ is reported in each plot.
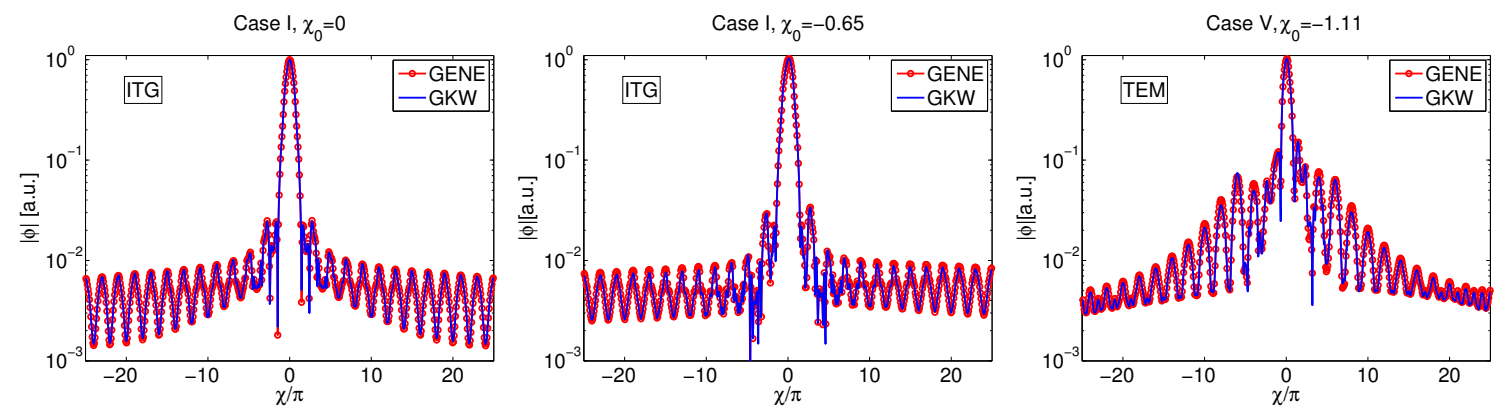

Figure 11. Same as Figure 10, but for Case I. 


\section{ROSENBLUTH - HINTON TEST}

Correctly describing Zonal Flows is essential for any turbulence simulation as they are one of the main mechanisms of saturation in non-linear regime, at least in ITG dominated plasmas. The Rosenbluth-Hinton test ${ }^{30}$ allows to study linear dynamics of ZFs by computing the residual level of e.g. the potential and at the same time characterize the properties (real frequency $\omega_{\mathrm{GAM}}$ and damping $\gamma_{\mathrm{GAM}}$ ) of the Geodesic Acoustic Mode $(\mathrm{GAM})^{31}$. Several theoretical works are available providing estimates for both the residual level and the GAM properties under various limits. Thus in addition to code benchmarking it is also possible to validate simulation results against such estimates, in their proper limit.

In order to avoid the numerical problem of small recurrence time due to light electrons, this particular test has been carried out considering the adiabatic response of the electrons. Fully kinetic simulations show the same level of residual potential, confirming the validity of the approach.

GENE simulations have been performed evolving an ion density perturbation associated to the mode $k_{x} \rho_{i}=0.05, k_{y}=0$ and solved on grids involving up to $n_{z} \times n_{v} \times n_{\mu}=$ $64 \times 400 \times 32$ points. No hyperdiffusion has been used in order to avoid any effect on the frequency of the GAM. ${ }^{32}$ Density and temperature gradients have been set to zero. The same set up was used for carrying out GKW simulations, which have been performed using $n_{s} \times n_{v_{\|}} \times n_{\mu}=140 \times 256 \times 27$ grid points. Hyperdiffusion has been switched off as well. GS2 adopts a different initial condition for Zonal Flow investigations, consisting in evolving in time an initial zonal electrostatic field without initiating any density perturbation. These different initial conditions are found to lead to the same final results. The runs performed with GS2 have been carried out considering $n_{\theta} \times n_{\lambda} \times n_{\mathcal{E}}=64 \times 32 \times 48$ grid points.

\section{A. Zonal Flow residual potential}

We benchmark the value of the residual potential $\langle\phi(\infty)\rangle /\langle\phi(0)\rangle$, defined as the flux surface averaged electrostatic potential $\langle\phi\rangle$ normalized to its initial value $\langle\phi(0)\rangle$. This quantity is computed after the GAM oscillation is completely damped. Simulations are run well beyond this limit, typically up to $150 R_{0} / v_{i}$, to ensure a true stationary state and check that the recurrence problem is not affecting the results. The obtained residual levels computed 
with GENE, GKW and GS2, are shown in Figure 12, and compared with several theoretical estimates available in the literature. These are all of the form

$$
\frac{\langle\phi(\infty)\rangle}{\langle\phi(0)\rangle}=\frac{1}{1+S q_{s}^{2} / \sqrt{\epsilon}}
$$

where $S$ is a shaping function dependent on the model used for describing the magnetic geometry. For circular concentric magnetic surfaces in large aspect ratio Tokamaks, Eq. (11)

reduces to the well known expression by Rosenbluth and Hinton $1 /\left(1+1.6 q_{s}^{2} / \sqrt{\epsilon}\right)$ valid to first order in $\epsilon$. For shaped Tokamaks, Xiao and $\mathrm{Catto}^{33}$ derived a shaping function valid up to second order in $\epsilon$ in which all shaping parameters explicitly appear

$$
\begin{gathered}
S_{X C}=\frac{1}{1+\kappa^{2}}\left(3.27+\sqrt{\epsilon}+0.722 \epsilon-1.44 \delta-2.945 \frac{\Delta}{\epsilon}\right. \\
\left.+\frac{0.692 k^{2}-0.722}{q_{s}^{2}} \epsilon\right)
\end{gathered}
$$

while Zhou and $\mathrm{Yu}^{34}$ adopted a Miller equilibrium to obtain

$$
\begin{aligned}
S_{Z Y}= & \frac{1}{\kappa^{2}(1+3 \delta \epsilon / 8)^{2} I_{0}}\left\{\frac{25}{16}-\frac{53}{256} \delta+\frac{\sqrt{\epsilon}}{2}-\epsilon\right. \\
& \left.\times\left[\frac{3}{64}-\frac{93}{256} \delta+\frac{9 \kappa^{2}}{8 q_{s}^{2}}\left(\frac{3 I_{0}}{4}+I_{1}\right)\right]\right\}
\end{aligned}
$$

$I_{0}$ and $I_{1}$ are two geometrical quantities defined in Ref. $34, q_{s}, \epsilon, \kappa, \delta$ and $\Delta$ are the local values at $q=q_{s}$. We note that the definition of $\epsilon$, elongation $\kappa$ triangularity $\delta$ and Shafranov shift $\Delta$ appearing in equations (12) and (13), depend on the particular parametrization used for describing the flux surface. Therefore their value is computed by fitting the CHEASE equilibrium accordingly to each specific magnetic geometry model.

The agreement obtained between the codes is very good, within $1 \%$ and independent from shaping. A systematic deviation from analytic estimates is found in all shaped cases (Case I to IV), among which the one by Zhou and Yu (Eq. (13)) is found to give the estimate closest to our numerical simulation $(\approx 10 \%$ lower $)$.

\section{B. GAM properties}

When performing the Rosenbluth-Hinton test, the GAM is excited and its real frequency and damping can be extracted from the time trace of the simulated potential. The damping $\gamma_{\mathrm{GAM}}$ is obtained upon fitting the maxima and minima of the residual to an exponential envelope. The frequency is subsequently extracted via inverse Hilbert transform. An example 


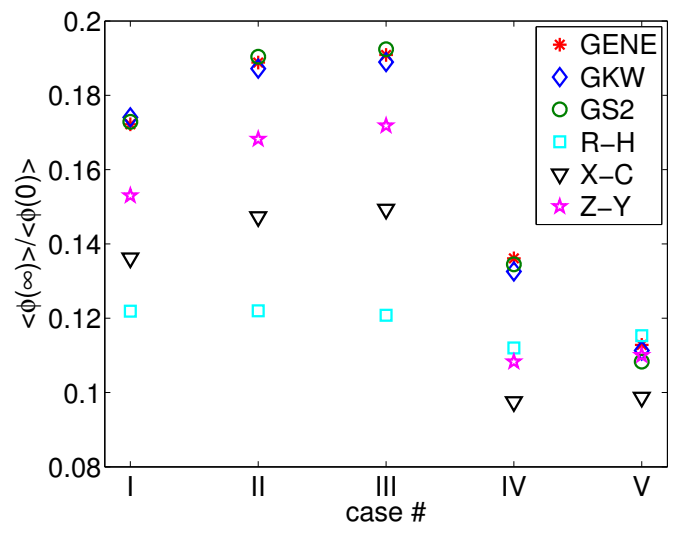

Figure 12. Residual potential computed with GENE (red stars), GKW (blue diamonds) and GS2 (green circles) normalized to its initial value for each equilibrium Case, I to V. For comparison, the theoretical estimates given by Rosenbluth-Hinton ${ }^{30}(\mathrm{R}-\mathrm{H})$, Xiao-Catto ${ }^{33}$ (X-C) and Zhou-Yu ${ }^{34}$ (Z-Y) are shown as well with cyan squares, black triangles and magenta stars respectively (color online).

is shown in Figure 13. When comparing different codes the same time window is used. This is necessary especially when evaluating the damping which is strong in the cases considered here and therefore only few GAM oscillations are contained in the simulated time trace. The

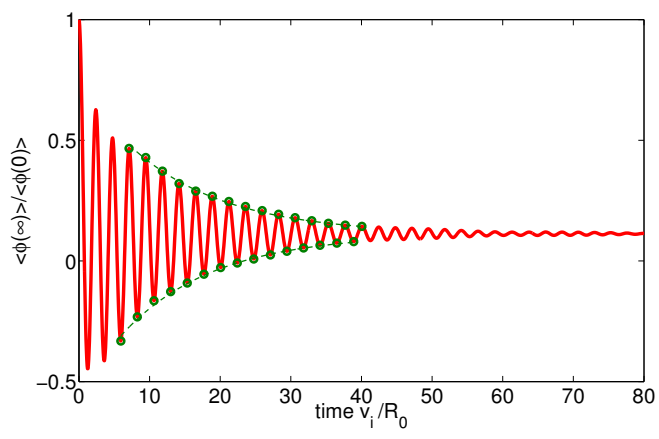

Figure 13. Time trace of electrostatic potential normalized to its initial value for Case V (values obtained with the GENE code). The points used to estimate GAM real frequency and damping are shown with green circles together with the corresponding estimated exponentially decaying envelope (color online).

three codes agree between each other on $\omega_{\text {GAM }}$ within $1 \%$. The numerical results, together with the numerical solution to the dispersions relations proposed in Refs. 35 and 36 are 
shown in Figure 14. The first analytical estimate, valid only for circular plasmas, agrees with the simulation only for Case $\mathrm{V}$, while the latter which retains shaping effects, matches the simulation in all Cases with less than 5\% difference. The strong GAM damping found in all shaped plasmas (Cases I to IV) makes its estimation from the simulation difficult. Nevertheless the codes agree between each other, while a sensible difference is found when comparing to analytical estimates.
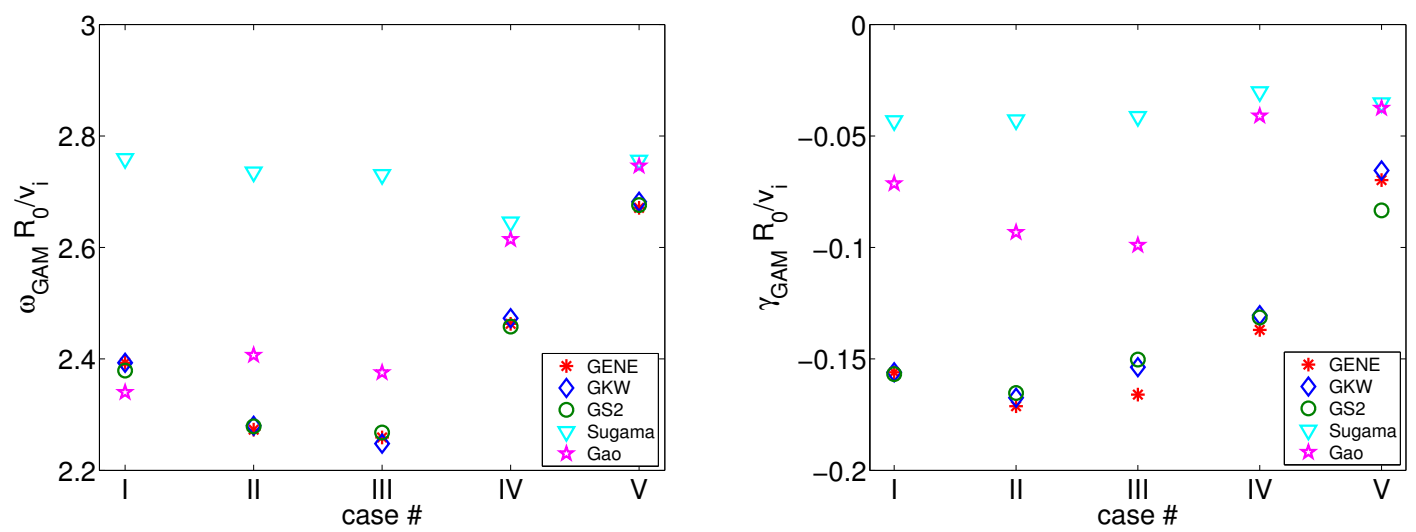

Figure 14. GAM real frequency $\omega_{\mathrm{GAM}}$ and damping rate $\gamma_{\mathrm{GAM}}$ for the five test Cases. Shown are GENE results (red stars), GKW ones (blue diamonds) and GS2 (green circles). The analytic predictions by Sugama ${ }^{35}$ (cyan triangles) and the one from $\mathrm{Gao}^{36}$ (magenta pentagrams) are shown as well (color online).

The same analysis as described in Section III of interfacing the flux surfaces with the Miller equilibrium for studying the effect of all parameters, has been repeated for the Rosenbluth-Hinton test. Figure 15, showing the residual potential level and the GAM properties, confirms that when going from one case to the other all the parameters play a role but whenever $\kappa$ is varied, it is responsible for the major part of the change. This is true in particular for Case II where, despite considering a triangular plasma, most of the difference is originated by a variation of elongation from Case II, and also for Case I. Note that in Fig. 15 the values have been normalized to $R_{\text {geom }}(r) / v_{i}$ for simplicity. We can therefore plot the results of this test with respect to elongation, as shown in Figure 16. The results are nicely 

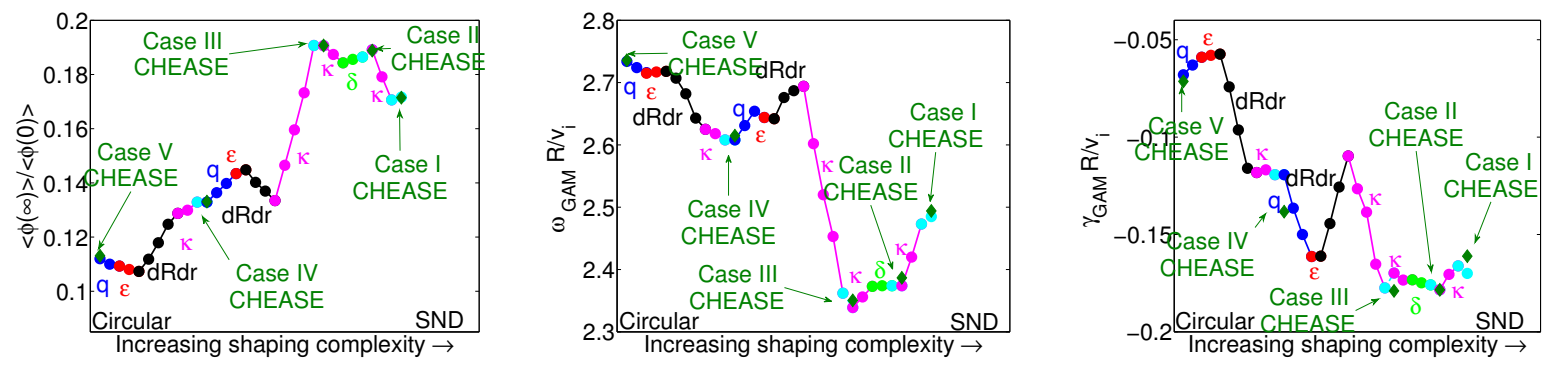

Figure 15. Effect of the shaping parameters on $(a)$ ZF residual, (b) GAM frequency $\omega_{\text {GAM }}$ and $(c)$ GAM damping $\gamma_{\text {GAM }}$. Results obtained with the GENE code performing the RH test after fitting the equilibria according the parametrization given by Eqs. (2) and (3). One parameter is varied at each time as indicated by labels, the values are listed in Table III. For comparison, the values obtained when using the CHEASE equilibrium are reported as well (green, color online).

aligned and show how an increase of $\kappa$ leads to an increase of the residual level while at the same time the GAM frequency is reduced and the mode is more strongly damped.
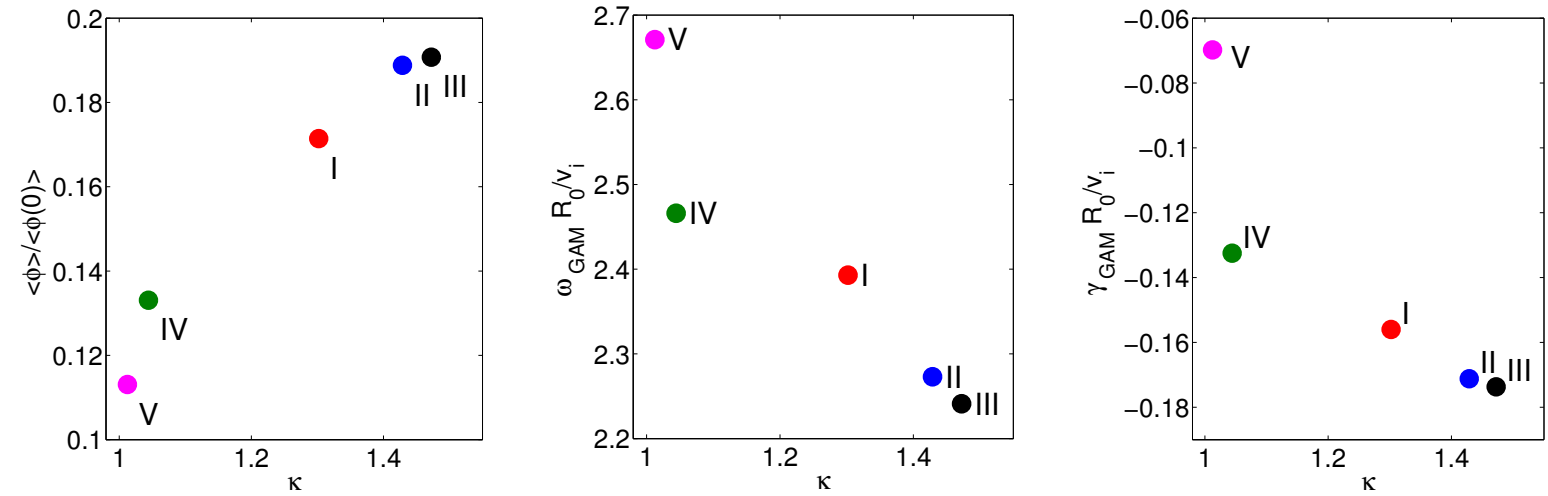

Figure 16. Results of the Rosenbluth-Hinton represented versus elongation $\kappa$ of each test Case. Shown are $(a)$ the residual level, $(b)$ the GAM real frequency $\omega_{G A M}$ and $(c)$ the damping $\gamma_{G A M}$. Numbers indicate the corresponding test case.

\section{CONCLUSIONS}

We have developed a series of benchmarks with the aim of testing the interface of gyrokinetic codes with kinetic ions and electrons and realistic shaped MHD equilibria, the latter being provided by the MHD equilibrium code CHEASE. These tests have been used to suc- 
cessfully benchmark against each other the three gyrokinetic codes GENE, ${ }^{12,13,26} \mathrm{GKW}^{14}$ and GS2 ${ }^{16}$ in the linear flux-tube limit. A fully gyrokinetic model for describing the electron dynamics was considered, while collisions and electromagnetic effects have been neglected. This exercise, which at first glance might appear trivial, requires in fact to pay attention to several subtleties that normally do not need to be faced when carrying out similar benchmarks but adopting circular analytic geometry. In particular, one has to be very careful regarding the particular choice of coordinates used within each code, not only to correctly define the location of the flux tube volume, but also because it determines how to correctly recast the results in a common representation for comparison. We have put a specific effort in writing this paper to provide all the details that have to be taken into account such as any other code can undergo the same benchmarks without uncertainties. All codes inputs and outputs, together with all useful information, are made public available in Ref. 19 for any other code interested in carrying out the same series of tests.

Several benchmarks have successfully been carried out looking at linear $k_{\theta}$ spectra, at the effect of a finite ballooning angle and studying the linear dynamics of ZFs and GAMs via the standard Rosenbluth-Hinton test. In all the tests that we have performed, the codes agree within $3 \%$, a difference that can be further reduced by properly increasing the resolution. This benchmark cannot be considered an exhaustive study of plasma shaping effects, but nevertheless it has demonstrated that among the parameters that we have considered, elongation plays the strongest stabilizing role and at the same time it increases the ZF residual level and reduces GAM frequency.

The natural extension of this benchmark exercise is towards global simulations. The equilibria that we have considered have already been designed for carrying out this kind of simulations and are explicitly built such that local and global results can be compared. We recognize that carrying out the same exercise, with possibly also non-linear global simulations, is a long and computationally expensive effort. However, we consider this as one of the fundamental next steps to be undertaken in order to validate gyrokinetic codes. 


\section{ACKNOWLEDGMENTS}

Simulations have been carried out using the HPC-FF cluster of the Jülich Forschungszentrum, the Monte Rosa CRAY XE-6 platform of the Swiss National Supercomputing Center (CSCS) and the HELIOS supercomputer system at the Computational Simulation Centre of International Fusion Energy Research Centre (IFERC-CSC), Aomori, Japan. This project has received funding from the European Union's Horizon 2020 research and innovation programme under grant agreement number 633053. The views and opinions expressed herein do not necessarily reflect those of the European Commission. This work was supported in part by the Swiss National Science Foundation and partially carried out with funding from the RCUK Energy Programme [grant number EP/I501045].

\section{Appendix A: The GENE code}

\section{GENE coordinate system}

The GENE code ${ }^{12,13,26}$ uses a field-aligned coordinate system $(x, y, z)$, expressed in terms of the magnetic straight field line coordinate system $(\psi, \chi, \varphi)$ as follows:

$$
\left\{\begin{array}{l}
x-x_{0}=C_{x}(\psi), \\
y=C_{y}[q(\psi) \chi-\varphi], \\
z=\chi
\end{array}\right.
$$

Here, $\psi$ is the poloidal flux function $\psi=(2 \pi)^{-1} \int_{V} \mathbf{B} \cdot \nabla \theta d^{3} x$ where $V$ is the volume enclosed by a magnetic surface, $\chi$ is the straight field-line poloidal angle, defined in terms of the geometrical poloidal angle $\theta$ as

$$
\begin{aligned}
\chi & =2 \pi \int_{0}^{\theta} \frac{\mathbf{B} \cdot \nabla \varphi}{\mathbf{B} \cdot \nabla \theta^{\prime}} d \theta^{\prime} / \oint \frac{\mathbf{B} \cdot \nabla \varphi}{\mathbf{B} \cdot \nabla \theta^{\prime}} d \theta^{\prime} \\
& =2 \pi \int_{0}^{\theta} \frac{1}{R^{2}} \frac{1}{\mathbf{B} \cdot \nabla \theta^{\prime}} d \theta^{\prime} / \oint \frac{1}{R^{2}} \frac{1}{\mathbf{B} \cdot \nabla \theta^{\prime}} d \theta^{\prime} \\
& =\frac{F(\psi)}{q(\psi)} \int_{0}^{\theta} \frac{1}{R^{2}} \frac{1}{\mathbf{B} \cdot \nabla \theta^{\prime}} d \theta^{\prime},
\end{aligned}
$$

and $\varphi$ is the toroidal angle.

In the GENE coordinate system, the $x$ coordinate defines the radial direction, while $y$ is called the binormal direction. Finally, $z$ parametrizes the position along a field line and 
therefore is referred to as the "parallel" direction. It is to be noted that in practice the choice of the radial coordinate $x$ is dependent on the magnetic equilibrium used: when interfacing with CHEASE equilibria $x=\rho_{\text {tor }}=\sqrt{\phi_{\text {tor }} / \pi B_{0}}$ is normally considered, $\phi_{t o r}=$ $(2 \pi)^{-1} \int_{V} \mathbf{B} \cdot \nabla \varphi d^{3} x$ being the toroidal flux, while when interfacing to Miller's equilibria $x=r$ is assumed. $C_{y}$ is a normalization constant, chosen as $C_{y}=x_{0} / q_{s}$ in the flux tube version of the code in order to establish $y$ as a length rather than an angle-like quantity. $q_{s}=q\left(x_{0}\right)$ indicates the local safety factor evaluated at $x=x_{0}$, the reference magnetic flux surface for the flux tube simulations. Note that both $(\psi, \chi, \varphi)$ and $(x, y, z)$ are nonorthogonal coordinate systems.

The magnetic field $\mathbf{B}$ can be written with respect to either $(\psi, \chi, \varphi)$ or $(x, y, z)$ as follows

$$
\mathbf{B}=F \nabla \varphi+\frac{1}{2 \pi} \nabla \varphi \times \nabla \psi=\mathcal{C}(\nabla x \times \nabla y)
$$

where $F(\psi)=R B_{\varphi}$, with $B_{\varphi}$ the toroidal component of the magnetic field, and $\mathcal{C}=1 /\left[2 \pi\left(d C_{x} / d \psi\right) C_{y}\right]$. It is clear from Eq. A3 that $\mathbf{B} \cdot \nabla x=\mathbf{B} \cdot \nabla y=0$, so that $x=$ const and $y=$ const define a magnetic field line, and $(x, y, z)$ indeed defines a field-aligned coordinate system.

In the flux tube version of the code, any fluctuating field quantity $A(x, y, z)$ (e.g. $A=\phi$, the electrostatic potential) is Fourier transformed with respect to both $x$ and $y$. In $(x, y, z)$ coordinates, axisymmetry translates to invariance with respect to $y$, so that linear fluctuation eigenmodes have an exact wave-number $k_{y}$ with respect to $y$. The corresponding Fourier representation therefore reads

$$
A(x, y, z)=e^{\mathrm{i} k_{y} y} \sum_{k_{x}} \hat{A}_{k_{x}}(z) e^{\mathrm{i} k_{x} x} \sim e^{-\mathrm{i} n \varphi}
$$

yielding together with Eq. (A1) the relation between $k_{y}$ and the toroidal mode number $n$ :

$$
n=k_{y} C_{y} \quad k_{y}=\frac{n}{C_{y}}=\frac{n q_{s}}{x_{0}} \sim k_{\theta},
$$

where $k_{\theta}$ is an estimate of the effective poloidal wave number for field-aligned fluctuations. Again using Eq. (A1), periodicity with respect to the toroidal direction $A(\psi, \chi, \varphi+2 \pi)=$ $A(\psi, \chi, \varphi)$ translates in $(x, y, z)$ coordinates to periodicity in the $y$ direction:

$$
A(x, y, z)=A\left(x, y+2 \pi C_{y}, z\right)
$$

It should be noted that flux-tube simulation volumes do not necessarily account for the full toroidal angle, so that the simulation box length $L_{y}$ does not necessarily coincides with $2 \pi C_{y}$. 
One nonetheless imposes periodicity in $y$, which therefore in general reads $A(x, y x, z)=$ $A\left(x, y+L_{y}, z\right)$. In turn, periodicity in the poloidal direction $A(\psi, \chi+2 \pi, \varphi)=A(\psi, \chi, \varphi)$ implies the following in $z$ direction:

$$
A(x, y, z+2 \pi)=A\left(x, y-2 \pi q C_{y}, z\right) .
$$

As a result of Eq. (A7), and after linearizing the safety factor profile, $q(x)=q_{s}\left(1+\hat{s} x / x_{0}\right)=$ $q_{s} \hat{s} / x_{0}\left(x+x_{0} / \hat{s}\right)$ around the reference position $x_{0}$, one can show that for a given $\delta k_{x}$ and $k_{y}$, the set of radial Fourier components

$$
k_{x}=p \Delta k_{x}+\delta k_{x}, \quad p \in \mathbb{Z},
$$

are coupled together, with $\Delta k_{x}=2 \pi \hat{s} k_{y}$, such that any linear fluctuation mode in the flux-tube limit reads:

$$
\begin{aligned}
& A(x, y, z)=e^{\mathrm{i} k_{y} y} e^{\mathrm{i} \delta k_{x} x} \sum_{p} \hat{A}_{\delta k_{x}+p \Delta k_{x}}(z) e^{\mathrm{i} p \Delta k_{x} x}, \\
& \text { with } \quad \hat{A}_{\delta k_{x}+p \Delta k_{x}}(z+2 \pi)=\hat{A}_{\delta k_{x}+(p+1) \Delta k_{x}}(z) .
\end{aligned}
$$

In deriving Eq. (A9), it has been assumed that the phase factor $e^{\mathrm{i} 2 \pi n q_{s}\left(x_{0}\right)}=1$, which in fact corresponds to centering the simulation domain around the mode rational surface nearest to $x_{0}$ for the considered $k_{y}=n / C_{y}$ (the distance between two neighboring rational surfaces $\Delta_{\text {MRS }}$ being $\left.1 / k_{y} \hat{s}\right)$.

\section{Ballooning angle}

The ballooning representation of a given fluctuating field $\mathcal{A}(x, \chi, \varphi)$ in straight field line coordinate system reads

$$
\mathcal{A}(x, \chi, \varphi)=\hat{\mathcal{A}}(\chi) e^{-i n\left\{\varphi-q(x)\left[\chi-\chi_{0}\right]\right\}} .
$$

The exponential term in (A10) represents the fast phase factor for field-aligned fluctuations, while $\chi_{0}$ corresponds to the so-called ballooning angle, angle at which the fast phase does not vary radially through $q(x) . \hat{\mathcal{A}}(\chi)$ is the so-called ballooning envelope and accounts for the slow variation of the fluctuation along the magnetic field line. Any radial modulation of the envelope has already been neglected. Equation (A10) does not ensure periodicity with 
respect to $\chi$ which is therefore enforced by expressing the actual field $A(x, \chi, \varphi)$ in terms of $\mathcal{A}(x, \chi, \varphi)$ as follows:

$$
\begin{aligned}
A(x, \chi, \varphi) & =\sum_{p=-\infty}^{+\infty} \mathcal{A}(x, \chi+p 2 \pi, \varphi) \\
& =e^{-\mathrm{i} n[\varphi-q(x) \chi]} \sum_{p=-\infty}^{+\infty} \hat{\mathcal{A}}(\chi+p 2 \pi) e^{\mathrm{i} n q(x)\left[p 2 \pi-\chi_{0}\right]}
\end{aligned}
$$

Identifying $k_{y} y=-n[\varphi-q(x) \chi]$ according to Eqs. (A1) and (A5), and after again linearizing the safety factor profile, one obtains

$$
\begin{aligned}
A(x, \chi, \varphi) & =e^{\mathrm{i} k_{y} y} \sum_{p=-\infty}^{+\infty} \hat{\mathcal{A}}(\chi+p 2 \pi) e^{\mathrm{i} k_{y} \hat{s} x\left(p 2 \pi-\chi_{0}\right)}, \\
& =e^{\mathrm{i} k_{y} y} e^{-\mathrm{i} k_{y} \hat{s} \chi_{0} x} \sum_{p=-\infty}^{+\infty} \hat{\mathcal{A}}(\chi+p 2 \pi) e^{\mathrm{i} p 2 \pi \Delta_{k_{x}} x} .
\end{aligned}
$$

In deriving equation (A12) the radial coordinate has again been radially shifted, i.e. $x+x_{0} / \hat{s} \rightarrow x$. Comparing equation (A9) to (A12), one thus identifies

$$
\left\{\begin{array}{l}
\chi_{0}=-\frac{\delta k_{x}}{k_{y} \hat{s}}, \\
\hat{\mathcal{A}}(\chi+p 2 \pi)=\hat{A}_{\delta k_{x}+p \Delta k_{x}}(z) .
\end{array}\right.
$$

\section{Normalization}

The normalization in the GENE code is chosen such that all dimensionless quantities are of order unity. Therefore, in configuration space independent variables $x$ and $y$ used for representing fluctuating fields are normalized to a reference gyroradius $\rho_{\text {ref }}$, while the already dimensionless field connection length along $z$ is kept to be $2 \pi \sim \mathcal{O}(1)$. A macroscopic length $L_{\text {ref }}$ is used to normalize gradients of equilibrium quantities. In velocity space however, in order to account for potentially differing temperatures, the normalization has to be species dependent. Furthermore magnetic fields, temperatures, densities and masses are normalized with respect to the reference values $B_{\text {ref }}, T_{\text {ref }}, n_{\text {ref }}$ and $m_{\text {ref }}$. The user is free to specify these reference values. Derived reference quantities such as the sound velocity $c_{\text {ref }}$ and the Larmor frequency $\Omega_{\text {ref }}$ and radius $\rho_{\text {ref }}$ are defined as

$$
c_{\mathrm{ref}}=\sqrt{T_{\mathrm{ref}} / m_{\mathrm{ref}}}, \quad \Omega_{\mathrm{ref}}=e B_{\mathrm{ref}} / m_{\mathrm{ref}}, \quad \rho_{\mathrm{ref}}=\frac{c_{\mathrm{ref}}}{\Omega_{\mathrm{ref}}} .
$$


Indicating the normalized quantities with a tilde, one has for the $j$-th species

$$
\begin{gathered}
x=\tilde{x} \rho_{\text {ref }}, \quad y=\tilde{y} \rho_{\text {ref }}, \quad z=\tilde{z}, \\
v_{\|, j}=\tilde{v}_{\|} \tilde{v}_{\text {th }, j} c_{\text {ref }}, \quad \mu_{j}=\tilde{\mu} \tilde{T}_{j} \frac{T_{\text {ref }}}{B_{\text {ref }}}, \quad t=\tilde{t} \frac{L_{\text {ref }}}{c_{\text {ref }}},
\end{gathered}
$$

where the thermal velocity $v_{\mathrm{th}, j}$ of the $j$-species is defined as

$$
v_{\mathrm{th}, j}=\sqrt{2 T_{j} / m_{j}}
$$

Applying these normalization rules to Eq. (A4) one obtains:

$$
\tilde{A}(\tilde{x}, \tilde{y}, \tilde{z})=e^{\mathrm{i} \tilde{k}_{y} \tilde{y}} \sum_{\tilde{k}_{x}} \hat{\tilde{A}}_{\tilde{k}_{x}}(\tilde{z}) e^{\mathrm{i} \tilde{k}_{x} \tilde{x}}
$$

We note that typical choices are $T_{\text {ref }}=T_{e}, n_{\text {ref }}=n_{e}$ and $m_{\text {ref }}=m_{i}$, such that $c_{\text {ref }}=c_{s}$, while $B_{\text {ref }}$ is typically the magnetic field on axis, $B_{\text {ref }}=B_{\text {mag }}$. The macroscopic distance $L_{\text {ref }}$ is usually taken to be either the major radius $R_{0}$ or the minor radius $a$ of the Tokamak.

\section{Appendix B: The GKW code}

\section{GKW coordinate system}

The GKW $\operatorname{code}^{14}$ employs a field aligned coordinate system $(r, \zeta, s)$ similar to the $(x, y, z)$ system considered in GENE. The $r$ coordinate corresponds to the geometric minor radius $r=\left(R_{\max }-R_{\min }\right) / 2$, while $\zeta$ is equivalent to the $y$ coordinate in GENE defined in Eq. (A1) within a scaling factor $2 \pi C_{y}$ :

$$
\zeta=\frac{1}{2 \pi}[q(\bar{\psi}) \chi-\varphi]=\frac{1}{2 \pi C_{y}} y,
$$

Similar to Eq. (A3), the magnetic field can thus be written as

$$
\mathbf{B}=F(\bar{\psi}) \nabla \varphi+\nabla \varphi \times \nabla \bar{\psi}=2 \pi \frac{d \bar{\psi}}{d r} \nabla r \times \nabla \zeta
$$

Comparing Eq. (A3) to (B2) one notes that the definitions for the poloidal magnetic fluxes $\psi$ and $\bar{\psi}$ considered in GENE and GKW respectively differ by a factor $2 \pi, \bar{\psi}=\psi / 2 \pi$. In general, caution must be taken regarding the different definitions and orientations of the coordinates considered in various codes for representing the magnetic field. A detailed description of this issue is given in Ref. 37, together with practical indications for conversion 
between different choices characterized by a so-called COCOS value.

The "parallel" coordinate $s$ considered in GKW is however different from the straight field line angle poloidal angle $\chi$ in GENE. The $s$ coordinate is in fact one of the Hamada coordinates $(\bar{\psi}, s, \gamma)$, defined as $s=s(\bar{\psi}, \theta), \gamma=\gamma(\bar{\psi}, \theta, \varphi)$, and such that the corresponding contravariant component of the magnetic field

$$
B^{s}=\mathbf{B} \cdot \nabla s \quad B^{\gamma}=\mathbf{B} \cdot \nabla \gamma
$$

are flux functions, i.e. $B^{\gamma}=B^{\gamma}(\bar{\psi}), B^{s}=B^{s}(\bar{\psi})$.

From these conditions one can derive

$$
\begin{gathered}
s(\bar{\psi}, \theta)=\int_{0}^{\theta} \frac{d \theta^{\prime}}{\mathbf{B} \cdot \nabla \theta^{\prime}} / \oint \frac{d \theta^{\prime}}{\mathbf{B} \cdot \nabla \theta^{\prime}} \\
\gamma(\bar{\psi}, \theta, \varphi)=\frac{\varphi}{2 \pi}+g(\theta, r)
\end{gathered}
$$

and

$$
\begin{gathered}
B^{s}(\bar{\psi})=\left[\oint \frac{d \theta^{\prime}}{\mathbf{B} \cdot \nabla \theta^{\prime}}\right]^{-1}, \\
B^{\gamma}(\bar{\psi})=\frac{F}{2 \pi}\left\langle\frac{1}{R^{2}}\right\rangle,
\end{gathered}
$$

with

$$
g(\bar{\psi}, \theta)=\frac{F(\bar{\psi})}{2 \pi} \int_{0}^{\theta} \frac{d \theta^{\prime}}{\mathbf{B} \cdot \nabla \theta^{\prime}}\left[\left\langle\frac{1}{R^{2}}\right\rangle-\frac{1}{R^{2}}\right] .
$$

Here $\langle\cdot\rangle$ stands for the flux surface average defined by (1), which can in fact also be rewritten as

$$
\langle\mathcal{A}\rangle=\oint d s \mathcal{A}=\frac{\oint d \theta^{\prime} \frac{\mathcal{A}}{\mathbf{B} \cdot \nabla \theta^{\prime}}}{\oint \frac{d \theta^{\prime}}{\mathbf{B} \cdot \nabla \theta^{\prime}}}
$$

for a quantity $\mathcal{A} \neq \mathcal{A}(\bar{\psi})$. One notes that the safety factor can be expressed as

$$
q(\bar{\psi})=\frac{B^{\gamma}(\bar{\psi})}{B^{s}(\bar{\psi})}=\frac{F}{2 \pi} \oint \frac{1}{R^{2}} \frac{d \theta}{\mathbf{B} \cdot \nabla \theta} .
$$

One can also show that the $\zeta$ coordinate defined in Eq. (B1) can be written as

$$
\zeta=q(\bar{\psi}) s-\gamma
$$

from which one derives the relation between $s$ and $\chi$

$$
\chi=2 \pi\left[s-\frac{g}{q(\bar{\psi})}\right] .
$$


As in the flux-tube version of GENE, any fluctuating field quantity $A(r, \zeta, s)$ is Fourier transformed with respect to $r$ and $\zeta$. The corresponding Fourier representation therefore reads

$$
A(r, \zeta, s)=e^{\mathrm{i} k_{\zeta} \zeta} \sum_{k_{r}} \hat{A}_{k_{r}}(s) e^{\mathrm{i} k_{r} r} \sim e^{-\mathrm{i} n \varphi}
$$

yielding together with Eq. (B1)

$$
n=\frac{k_{\zeta}}{2 \pi}
$$

$2 \pi$-periodicity with respect to $\chi$ in $(\bar{\psi}, \chi, \varphi)$ coordinates translates in $(r, \zeta, s)$ coordinates to the pseudo-periodic condition with respect to $s$ :

$$
A(r, \zeta, s+1)=A(r, \zeta-q(r), s)
$$

For a given $k_{\zeta}$ mode, this condition leads to coupling between the set of considered $k_{r}$ modes, $k_{r}=\delta k_{r}+p \Delta k_{r}$ with $\Delta k_{r}=k_{\zeta} d q / d r$, so that Eq. (B13) becomes, after having furthermore linearized the safety factor

$$
\begin{gathered}
A(r, \zeta, s)=e^{\mathrm{i} k_{\zeta} \zeta} e^{\mathrm{i} \delta k_{r} r} \sum_{p=-\infty}^{+\infty} \hat{A}_{\delta k_{r}+p \Delta k_{r}}(s) e^{\mathrm{i} p \Delta k_{r} r}, \\
\hat{A}_{\delta k_{r}+p \Delta k_{r}}(s+1)=\hat{A}_{\delta k_{r}+(p+1) \Delta k_{r}}(s) .
\end{gathered}
$$

It is to be noted that when specifying the input parameters, $\left.k_{\theta}\right|_{\text {GKW }}$ is given instead of $k_{\zeta}$ itself. The value of $k_{\zeta}$ is then determined from $\left.k_{\theta}\right|_{\mathrm{GKW}}=\sqrt{g^{\zeta \zeta k_{\zeta}^{2}}}$ evaluated at the outer midplane $(s=0), g^{\zeta \zeta}=\nabla \zeta \cdot \nabla \zeta$ being the diagonal metric tensor related to $\zeta$. In a similar way, the value of $k_{r}$ is specified via $k_{R}=\sqrt{g^{r r} k_{r}^{2}}$.

In the same way as one derived Eq. (A12) from (A11), one can express the ballooning representation given in (A12) in terms of GKW-specific variables:

$$
\begin{aligned}
A(r, \zeta, s) & =e^{\mathrm{i} k_{\zeta} \zeta} \sum_{p=-\infty}^{+\infty} \hat{\mathcal{A}}(\chi+p 2 \pi) e^{\mathrm{i} \frac{k_{\zeta}}{2 \pi} \frac{d q}{d r}\left(r+\frac{r_{0}}{\hat{s}}\right)\left(p 2 \pi-\chi_{0}\right)} \\
& =e^{\mathrm{i} k_{\zeta} \zeta} e^{-\mathrm{i} \frac{k_{\zeta} \frac{d q}{d r} \chi_{0}}{2 \pi}} \sum_{p=-\infty}^{+\infty} \hat{\mathcal{A}}(\chi+p 2 \pi) e^{\mathrm{i} p \Delta k_{r} r \frac{d q}{d r}}
\end{aligned}
$$

having again shifted the radial coordinate $r+r_{0} / \hat{s} \rightarrow r$. Comparing Eq. (B17) to Eq. (B16) one can identify

$$
\left\{\begin{array}{l}
\chi_{0}=-2 \pi \frac{\delta k_{r}}{k_{\zeta} \frac{d q}{d r}} \\
\hat{\mathcal{A}}(\chi+p 2 \pi)=\hat{A}_{\delta k_{r}+p \Delta k_{r}}(s) .
\end{array}\right.
$$




\section{Normalization}

As in the GENE code, all quantities are normalized to be order unity and again speciesdependent normalization factors are adopted for the velocity space. The specific choices are however different, and are discussed here. In the GKW code, reference mass $m_{\text {ref }}$, density $n_{\text {ref }}$, temperature $T_{\text {ref }}$ and major radius $R_{\text {ref }}$ are defined, and the user is free to choose their value. When interfacing with the CHEASE code, $R_{\text {rref }}=R_{\text {geom }}(L C F S)$ it is assumed and the reference value of the magnetic field $B_{\text {ref }}$ is evaluated at the same location. These quantities are related via the definition of the reference thermal velocity $v_{\text {th,ref }}$

$$
v_{\mathrm{th}, \mathrm{ref}}=\sqrt{\frac{2 T_{\mathrm{ref}}}{m_{\mathrm{ref}}}}
$$

and used to compute the reference gyroradius $\rho_{\text {ref }}=v_{\text {th,ref }} / \Omega_{\text {ref }}$. These reference values are then used to compute for each species a dimensionless mass $\tilde{m}$, thermal velocity $\tilde{v}$, density $\tilde{n}$ and temperature $\tilde{T}$

$$
\tilde{m}=\frac{m}{m_{\text {ref }}}, \quad \tilde{v}=\frac{v_{\text {th }}}{v_{\text {th,ref }}}, \quad \tilde{n}=\frac{n}{n_{\text {ref }}}, \quad \tilde{T}=\frac{T}{T_{\text {ref }}} .
$$

With these choices, the GKW phase space coordinates are all normalized according to

$$
\begin{gathered}
r=\tilde{r} R_{\mathrm{ref}}, \quad \zeta=\tilde{\zeta}, \quad s=\tilde{s} \\
v_{\|, j}=\tilde{v}_{\|} v_{\mathrm{th}, \mathrm{j}}, \quad \mu=\tilde{\mu} \frac{m v_{\mathrm{th}, \mathrm{j}}^{2}}{B_{\mathrm{ref}}}, \quad t=\tilde{t} \frac{R_{\mathrm{ref}}}{v_{\mathrm{th}, \mathrm{ref}}},
\end{gathered}
$$

where $v_{\mathrm{th}, \mathrm{j}}$ is the thermal velocity of the $j$-species as defined in Eq.(B19).

We explicitly remark the different definition of the reference velocity with respect to what is done in the GENE code, which is responsible, within a factor $\sqrt{2}$, for a different normalization of the microscopic scales quantities as well as of time. On the other hand, the normalized velocity space variables are the same. Furthermore, the normalization of the radial direction with respect to the macroscopic length $R_{\text {ref }}$ causes a factor $\bar{\rho}=\rho_{\text {ref }} / R_{\text {ref }}$ to explicitly appear in the normalized equivalent of the Fourier representation given in e.g. Eq. (B13) which then reads

$$
\tilde{A}(\tilde{r}, \tilde{\zeta}, \tilde{s})=e^{\frac{\mathrm{i} \tilde{k}_{\zeta} \tilde{\zeta}}{\bar{\rho}}} \sum_{\tilde{k}_{r}} \hat{\tilde{A}}_{\tilde{k}_{r}}(\tilde{s}) e^{\frac{\mathrm{i} \tilde{k}_{r} \tilde{r}}{\bar{\rho}}}
$$




\section{Appendix C: The GS2 code}

\section{GS2 coordinate system}

The GS2 code $^{16}$ employes a Clebsch formulation to express the magnetic field as

$$
\mathbf{B}=\nabla \alpha \times \nabla \psi
$$

where $\psi$ is the poloidal flux function, defined as in the GENE code. The function $\alpha=\alpha(\psi, \theta, \varphi)$ is determined by comparing Eq. (C1) to the equivalent representation $\mathbf{B}=F(\psi) \nabla \varphi+\nabla \varphi \times \nabla \psi$ as

$$
\alpha=\varphi-q \chi
$$

The GS2 field-aligned coordinate system $(X, Y, \theta)$ is directly based on the function $\alpha$ defined in Eq. (C2). In particular it is assumed for the radial direction $X$

$$
X=\frac{q_{s}}{r_{0} B_{a}} \psi
$$

while for the binormal $Y$ coordinate

$$
Y=\frac{d \psi}{d r} \frac{\alpha}{B_{a}}
$$

where $B_{a}$ is the toroidal magnetic field measured at the geometric center of the flux surface of interest: $B_{a}=F\left(R_{\text {geom }}\right) / R_{\text {geom. }}$. We point out that in the GS2 code, the geometric poloidal angle $\theta$ is used to parametrize a field line, therefore defining a "parallel" direction equivalent to the $z$ coordinate in GENE or the $s$ direction in GKW. Also the binormal direction $Y$ is again equivalent, within a sign and different scaling factors, to both the $y$ coordinate defined in Eq. (A1) for the GENE code and the $\zeta$ coordinate defined in Eq. (B1) for the GKW code:

$$
y=-C_{y} B_{a} \frac{d r}{d \psi} Y
$$

and

$$
\zeta=-\frac{B_{a}}{2 \pi} \frac{d r}{d \psi} Y
$$

As for the GENE and GKW codes, fluctuating field quantities $A(X, Y, \theta)$ are Fourier transformed with respect to both the radial and binormal directions. The corresponding Fourier representation in GS2 variables therefore reads

$$
A(X, Y, \theta)=e^{\mathrm{i} k_{Y} Y} \sum_{k_{X}} \hat{A}_{k_{X}}(\theta) e^{\mathrm{i} k_{X} X} \sim e^{\mathrm{i} n \varphi}
$$


where we note the different sign appearing in the eikonal with respect to the ones in GENE and GKW representations given in equations (A4) and (B13) respectively, yielding together with Eq.(C4)

$$
n=\frac{1}{B_{a}} \frac{d \psi}{d r} k_{Y}
$$

The set of radial $k_{X}$ modes, $k_{X}=\delta k_{X}+p \Delta k_{X}$, with $\Delta k_{X}=2 \pi \hat{s} k_{Y}$, are again coupled as a consequence of $2 \pi$-periodicity with respect to $\chi$ in $(\psi, \chi, \varphi)$ coordinates Equation $(\mathrm{C} 7)$, after linearizing the safety factor, can thus be rewritten as

$$
\begin{gathered}
A(X, Y, \theta)=e^{\mathrm{i} k_{Y} Y} e^{\mathrm{i} \delta k_{X} X} \sum_{p=-\infty}^{+\infty} \hat{A}_{\delta k_{X}+p \Delta k_{X}}(\theta) e^{\mathrm{i} p \Delta k_{X} X}, \\
\hat{A}_{\delta k_{X}+p \Delta k_{X}}(\theta+2 \pi)=\hat{A}_{\delta k_{X}+(p+1) \Delta k_{X}}(\theta) .
\end{gathered}
$$

Proceeding as already explained in Appendix A 2 for the GENE code, one derives the ballooning representation given in Eq. (A12) in terms of GS2-specific variables:

$$
\begin{aligned}
A(X, Y, \theta) & =e^{\mathrm{i} k_{Y} Y} \sum_{p=-\infty}^{+\infty} \hat{\mathcal{A}}(\chi+p 2 \pi) e^{\mathrm{i} k_{Y} \hat{s}\left(X+\frac{X_{0}}{\hat{s}}\right)\left(p 2 \pi-\chi_{0}\right)} \\
& =e^{\mathrm{i} k_{Y} Y} e^{-\mathrm{i} k_{Y} \hat{s} \chi_{0} X} \sum_{p=-\infty}^{+\infty} \hat{\mathcal{A}}(\chi+p 2 \pi) e^{\mathrm{i} p \Delta k_{X} X}
\end{aligned}
$$

having once again shifted the radial coordinate $X+X_{0} / \hat{s} \rightarrow X$. Comparing Eq. (C10) to Eq. (C9) one finds:

$$
\left\{\begin{array}{l}
\chi_{0}=-2 \pi \frac{\delta k_{X}}{k_{Y} \hat{s}} \\
\hat{\mathcal{A}}(\chi+p 2 \pi)=\hat{A}_{\delta k_{X}+p \Delta k_{X}}(\theta) .
\end{array}\right.
$$

\section{Normalization}

In the GS2 code, one chooses a reference value for temperature, density and mass, respectively $T_{\text {ref }}, n_{\text {ref }}$ and $m_{\text {ref }}$, while the reference thermal velocity $v_{\text {th,ref }}$ is defined as $v_{\text {th,ref }}=\sqrt{2 T_{\text {ref }} / m_{\text {ref }}}$. These values are used to compute the reference Larmor radius and frequency $\rho_{\text {ref }}$ and $\Omega_{\text {ref }}$, where we note that the magnetic field $B_{\text {ref }}$ is in this case defined as the toroidal field at the location of geometric center of the flux surface of interest, $B_{\text {ref }}=B_{a}$. All the macroscopic lengths are normalized to the minor radius $a$. In the same way as in the GENE and GKW codes, the velocity space variables are species-dependent normalized. Therefore, the GS2 coordinate system can be written as

$$
X=\tilde{X} \rho_{\text {ref }}, \quad Y=\tilde{Y} \rho_{\text {ref }}, \quad \theta=\tilde{\theta}
$$




$$
\mathcal{E}=\tilde{\mathcal{E}} T_{j}, \quad \lambda=\tilde{\lambda} B_{\text {ref }}, \quad t=\tilde{t} \frac{v_{\text {th,ref }}}{a}
$$

We note that with the aforementioned choices, the reference Larmor radius is the same as in the GKW code, while a factor $\sqrt{2}$ remains compared to the GENE code. However time and gradients of equilibrium quantities, because of the different choice of the macroscopic reference length, are normalized to a different value with respect to both GENE and GKW.

\section{REFERENCES}

${ }^{1}$ D. Told, F. Jenko, T. Görler, F. J. Casson, E. Fable, and A. U. Team, Physics of Plasmas 20, $122312(2013)$.

${ }^{2}$ A. M. Dimits, G. Bateman, M. A. Beer, B. I. Cohen, W. Dorland, G. W. Hammett, C. Kim, J. E. Kinsey, M. Kotschenreuther, A. H. Kritz, L. L. Lao, J. Mandrekas, W. M. Nevins, S. E. Parker, A. J. Redd, D. E. Shumaker, R. Sydora, and J. Weiland, Physics of Plasmas 7, 969 (2000).

${ }^{3}$ Y. Chen, S. E. Parker, W. Wan, and R. Bravenec, Physics of Plasmas 20, 092511 (2013). ${ }^{4}$ X. Lapillonne, B. F. McMillan, T. Görler, S. Brunner, T. Dannert, F. Jenko, F. Merz, and L. Villard, Physics of Plasmas 17, 112321 (2010).

${ }^{5}$ G. L. Falchetto, B. D. Scott, P. Angelino, A. Bottino, T. Dannert, V. Grandgirard, S. Janhunen, F. Jenko, S. Jolliet, A. Kendl, B. F. McMillan, V. Naulin, A. H. Nielsen, M. Ottaviani, A. G. ers, M. J. Pueschel, D. Reiser, T. T. Ribeiro, and M. Romanelli, Plasma Physics and Controlled Fusion 50, 124015 (2008).

${ }^{6}$ R. V. Bravenec, J. Candy, M. Barnes, and C. Holland, Physics of Plasmas 18, 122505 (2011).

${ }^{7}$ R. V. Bravenec, Y. Chen, J. Candy, W. Wan, and S. Parker, Physics of Plasmas 20, 104506 (2013).

${ }^{8}$ T. Görler, A. E. White, D. Told, F. Jenko, C. Holland, and T. L. Rhodes, Physics of Plasmas 21, 122307 (2014).

${ }^{9}$ D. R. Mikkelsen, M. Nunami, T.-H. Watanabe, H. Sugama, and K. Tanaka, Physics of Plasmas 21, 112305 (2014).

${ }^{10}$ S. Satake, Y. Idomura, H. Sugama, and T.-H. Watanabe, Computer Physics Communications 181, 1069 (2010). 
${ }^{11}$ P. W. Terry, M. Greenwald, J.-N. Leboeuf, G. R. McKee, D. R. Mikkelsen, W. M. Nevins, D. E. Newman, D. P. Stotler, T. G. on Verification, Validation, U. B. P. Organization, and U. T. T. Force, Physics of Plasmas 15, 062503 (2008).

${ }^{12}$ F. Jenko, W. Dorland, M. Kotschenreuther, and B. N. Rogers, Physics of Plasmas 7, 1904 (2000).

${ }^{13}$ See http://genecode.org/ for a description of the GENE code ().

${ }^{14}$ A. G. Peeters, Y. Camenen, F. J. Casson, W. A. Hornsby, A. P. Snodin, D. Strintzi, and G. Szepesi, Computer Physics Communications 180, 2650 (2009).

${ }^{15}$ See https://bitbucket.org/gkw/gkw/ for a description of the GKW code ().

${ }^{16}$ W. Dorland, F. Jenko, M. Kotschenreuther, and B. N. Rogers, Phys. Rev. Lett. 85, 5579 (2000).

${ }^{17}$ H. Lütjens, A. Bondeson, and O. Sauter, Computer Physics Communications 97, 219 (1996).

${ }^{18}$ A. Burckel, O. Sauter, C. Angioni, J. Candy, E. Fable, and X. Lapillonne, Journal of Physics: Conference Series 260, 012006 (2010).

${ }^{19}$ Inputs files required for the various benchmarks and code results are available at http: //crpp.epfl.ch/benchmark ().

${ }^{20}$ A. Marinoni, S. Brunner, Y. Camenen, S. Coda, J. P. Graves, X. Lapillonne, A. Pochelon, O. Sauter, and L. Villard, Plasma Physics and Controlled Fusion 51, 055016 (2009).

${ }^{21}$ R. L. Miller, M. S. Chu, J. M. Greene, Y. R. Lin-Liu, and R. E. Waltz, Physics of Plasmas 5, 973 (1998).

${ }^{22}$ J. Candy, Plasma Physics and Controlled Fusion 51, 105009 (2009).

${ }^{23}$ M. Kammerer, F. Merz, and F. Jenko, Physics of Plasmas 15, 052102 (2008).

${ }^{24}$ F. Merz, C. Kowitz, E. Romero, J. Roman, and F. Jenko, Computer Physics Communications 183, 922 (2012).

${ }^{25}$ J. E. Roman, M. Kammerer, F. Merz, and F. Jenko, Parallel Computing 36, 339 (2010), parallel Matrix Algorithms and Applications.

${ }^{26}$ F. Merz, Gyrokinetic simulations of multimode plasma turbulence, Ph.D. thesis, Universität Münster (2008).

${ }^{27}$ R. Singh, S. Brunner, R. Ganesh, and F. Jenko, Physics of Plasmas 21, 032115 (2014).

${ }^{28}$ Y. Camenen, Y. Idomura, S. Jolliet, and A. Peeters, Nuclear Fusion 51, 073039 (2011). 
${ }^{29}$ P. Migliano, Y. Camenen, F. J. Casson, W. A. Hornsby, and A. G. Peeters, Physics of Plasmas 20, 022101 (2013).

${ }^{30}$ M. N. Rosenbluth and F. L. Hinton, Phys. Rev. Lett. 80, 724 (1998).

${ }^{31}$ N. Winsor, J. L. Johnson, and J. M. Dawson, Physics of Fluids 11, 2448 (1968).

${ }^{32}$ J. Candy and R. E. Waltz, Physics of Plasmas 13, 032310 (2006).

${ }^{33}$ Y. Xiao, P. J. Catto, and W. Dorland, Physics of Plasmas 14, 055910 (2007).

${ }^{34}$ D. Zhou and Y. W., Physics of Plasmas 18, 052505 (2011).

${ }^{35}$ H. Sugama and T.-H. Watanabe, Journal of Plasma Physics 72, 825 (2006).

${ }^{36}$ Z. Gao, Physics of Plasmas 17, 092503 (2010).

${ }^{37}$ O. Sauter and S. Y. Medvedev, Computer Physics Communications 184 (2013). 